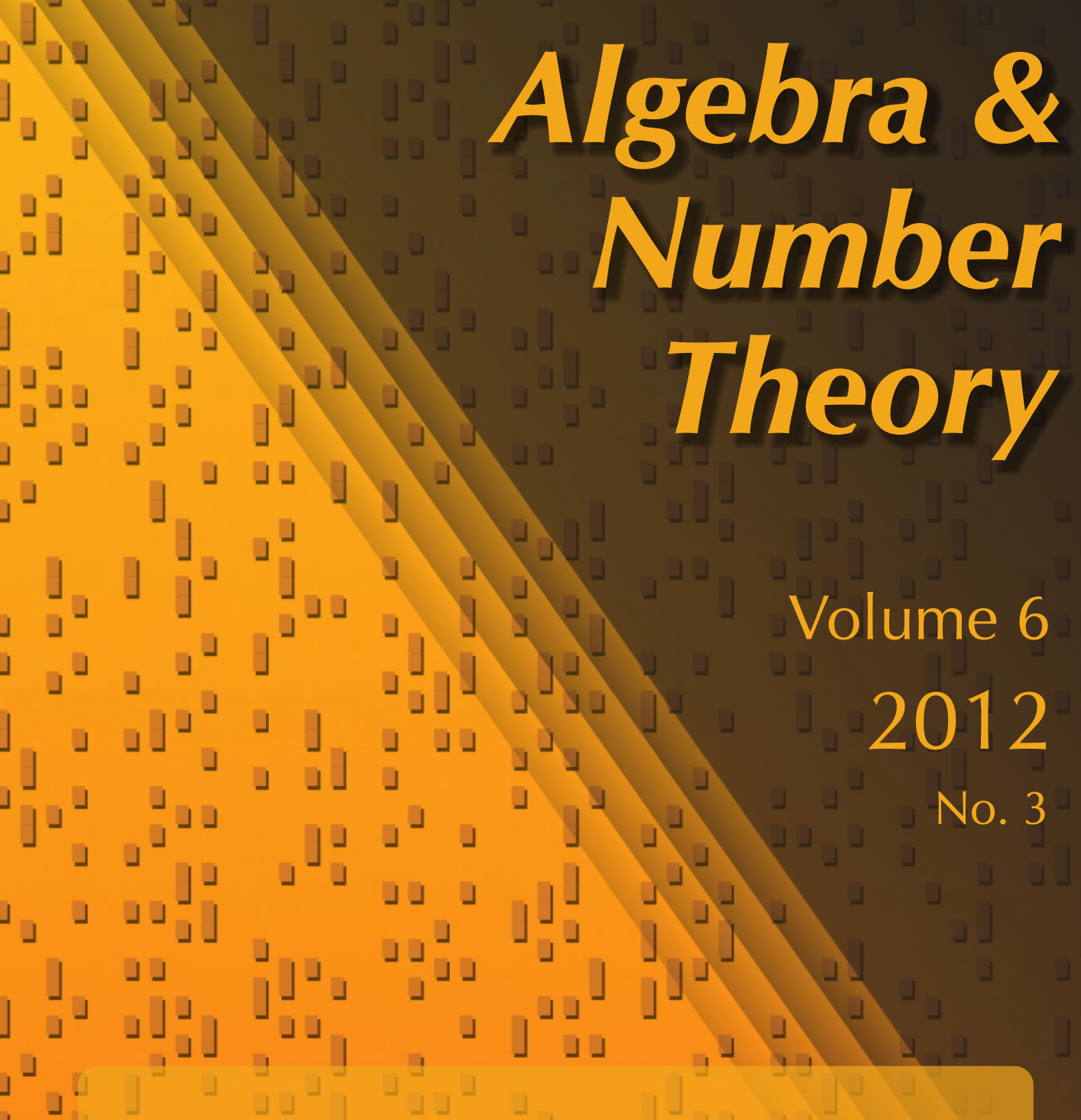

On the weak Lefschetz property for powers of linear forms

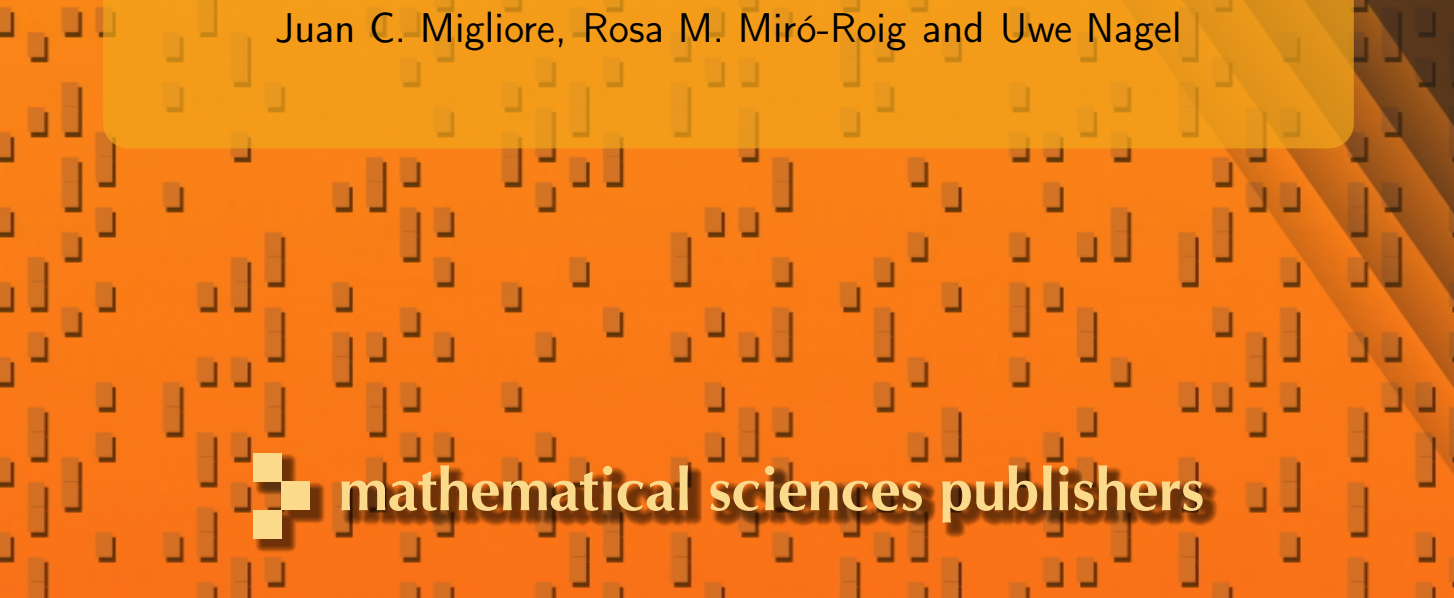




\title{
On the weak Lefschetz property for powers of linear forms
}

\author{
Juan C. Migliore, Rosa M. Miró-Roig and Uwe Nagel
}

Ideals generated by prescribed powers of linear forms have attracted a great deal of attention recently. In this paper we study properties that hold when the linear forms are general, in a sense that we make precise. Analogously, one could study so-called "general forms" of the same prescribed degrees. One goal of this paper is to highlight how the differences between these two settings are related to the weak Lefschetz property (WLP) and the strong Lefschetz property (SLP). Our main focus is the case of powers of $r+1$ general linear forms in $r$ variables. For four variables, our results allow the exponents to all be different, and we determine when the WLP holds and when it does not in a broad range of cases. For five variables, we solve this problem in the case where all the exponents are equal (uniform powers), and in the case where one is allowed to be greater than the others. For evenly many variables $(\geq 6)$ we solve the case of uniform powers, and in particular we prove half of a recent conjecture by Harbourne, Schenck and Seceleanu by showing that for evenly many variables, an ideal generated by $d$-th powers of $r+1$ general linear forms fails the WLP if and only if $d>1$. For uniform powers of an odd number of variables, we also give a result for seven variables, missing only the case $d=3$. Our approach in this paper is via the connection (thanks to Macaulay duality) to fat point ideals, together with a reduction to a smaller projective space, and the use of Cremona transformations.

1. Introduction 488

2. Lefschetz properties and Fröberg's conjecture 491

3. General approach 494

4. Powers of linear forms in four variables 497

5. Almost uniform powers of linear forms in 5 variables $\quad 514$

Part of the work for this paper was done while Migliore was sponsored by the National Security Agency under Grant Number H98230-09-1-0031.

Miró-Roig was partially supported by MTM2010-15256.

Part of the work for this paper was done while Nagel was sponsored by the National Security Agency under Grant Number H98230-09-1-0032.

MSC2010: primary 14C20, 13D40; secondary 13E10, 13C13, 13D02.

Keywords: weak Lefschetz property, artinian algebra, powers of linear forms, fat points. 
6. Uniform powers of linear forms

\section{Introduction}

Ideals generated by powers of linear forms have attracted a great deal of attention recently. For example, their Hilbert functions have been the focus of the papers [Ardila and Postnikov 2010; Sturmfels and Xu 2010; Harbourne et al. 2011], among others. In this paper we obtain further results in this direction, and relate them to the presence or failure of the weak Lefschetz property, which we now recall.

Let $A=R / I$ be a standard graded artinian algebra, where $R=k\left[x_{1}, \ldots, x_{r}\right]$ and $k$ is a field. If $\ell$ is a linear form, then multiplication by $\ell$ induces a homomorphism from any component $[A]_{i}$ to the next. Such linear forms are parametrized by $[R]_{1}$. A natural question is whether there is a Zariski-open subset $U$ of $[R]_{1}$ such that if $\ell$ corresponds to any point of $U$, then for each $i$, multiplication by $\ell$ induces a homomorphism of maximal rank. When this property holds, the algebra is said to have the weak Lefschetz property (WLP), and we say that multiplication by a general linear form has maximal rank from each degree to the next. One would naively expect this property to hold, and so it is interesting to find classes of algebras where it fails and to understand what is it about the algebra that prevents this property from holding. There has been a long series of papers by many authors studying different aspects of this problem. Even the characteristic of $k$ plays an interesting role; see for instance [Migliore et al. 2011; Li and Zanello 2010; Brenner and Kaid 2011; Cook and Nagel 2009; 2011].

The first result in this direction is due to R. Stanley [1980], J. Watanabe [1987], and L. Reid, L. Roberts and M. Roitman [Reid et al. 1991], who showed that, in characteristic 0, the WLP holds for an artinian complete intersection generated by powers of variables. In fact, they showed that there is a Zariski-open subset $U$ of $[R]_{1}$ such that if $\ell$ corresponds to a point of $U$, then for each $i$, multiplication by any power $\ell^{d}$ induces a homomorphism of maximal rank from $[A]_{i}$ to $[A]_{i+d}$; that is, the strong Lefschetz property (SLP) holds. Since both the WLP and the SLP are preserved after a change of variables, their result shows that both properties hold for any complete intersection whose generators are powers of linear forms. By semicontinuity, it holds for a complete intersection whose generators (of arbitrary degree) are chosen generically.

There are (at least) three natural directions suggested by this theorem. First, we can ask whether the WLP holds for arbitrary complete intersections. It was shown by T. Harima, J. Watanabe and the first and third authors in [Harima et al. 2003] that in two variables, all artinian algebras have the WLP. In the same paper, 
it was shown that it also holds for arbitrary artinian complete intersections in three variables. It remains open whether it also holds for arbitrary complete intersections in arbitrarily many variables.

Second, a natural question arising from the theorem of [Stanley 1980; Watanabe 1987; Reid et al. 1991] is to ask for which monomial ideals does the WLP hold or not hold. F. Zanello [2006] and H. Brenner and A. Kaid [2007] gave very simple examples to show that even level monomial ideals need not have this property, and the latter gave an example that was even an almost complete intersection (the ideal was in a ring with three variables and had four minimal generators). This latter fact gave a negative answer to a question in [Migliore and Miró-Roig 2003]. In [Migliore et al. 2011], we gave a much more extensive study of monomial almost complete intersections and when they fail to have the WLP. This work was extended in [Cook and Nagel 2009; 2011]. In [Boij et al. 2012], we showed that the only other situation where level monomial ideals have to have the WLP is 3 variables, type 2 .

A third interesting problem suggested by the result of [Stanley 1980; Watanabe 1987; Reid et al. 1991] is to ask when the WLP holds for powers of $\geq r+1$ linear forms, since up to a change of variables their result says that any complete intersection of powers of linear forms has the WLP. In [Migliore et al. 2011], we showed by example that in four variables, for $d=3, \ldots, 12$, an ideal generated by the $d$-th powers of five general linear forms does not have the WLP. On the other hand, H. Schenck and A. Seceleanu [2010] then gave the surprising result that in three variables, any ideal generated by powers of linear forms has the WLP. (We give a new proof of this result in Section 2.) In contrast, Harbourne, Schenck and Seceleanu [Harbourne et al. 2011] have recently shown the following: Let

$$
I=\left\langle\ell_{1}^{t}, \ldots, \ell_{n}^{t}\right\rangle \subset k\left[x_{1}, \ldots, x_{4}\right]
$$

with $\ell_{i}$ general linear forms. If $n \in\{5,6,7,8\}$ then the WLP fails, respectively, for $t \geq\{3,27,140,704\}$.

A famous conjecture of Fröberg [1985] gives the expected Hilbert function for an ideal of $s$ general forms of prescribed degrees $d_{1}, \ldots, d_{s}$. The result of [Stanley 1980; Watanabe 1987; Reid et al. 1991] shows that when $s=r+1$, the same Hilbert function is obtained by the same powers $d_{1}, \ldots, d_{r+1}$ of general linear forms. Many authors have studied the question of when an ideal of powers of general linear forms has the Hilbert function predicted by Fröberg, and it is known that often it fails even when $s=r+2$; see for instance [Iarrobino 1997]. It is also known (and strongly used in this paper) that there is a strong connection between the Hilbert function of powers of general linear forms and the Hilbert function of a related set of fat points in projective space. The connections between these topics, and a geometric study of the Hilbert function of a set of fat points via Bézout methods, can be found in [Iarrobino 1997] and in [2005; Chandler 2007]. 
In this paper, we study the WLP for quotients $k\left[x_{1}, \ldots, x_{r}\right] / I$ where $I$ is an almost complete intersection ideal generated by powers of general linear forms. By the main result of [Schenck and Seceleanu 2010], the first interesting case is $r=4$, and by the result of [Stanley 1980; Watanabe 1987; Reid et al. 1991], the first interesting situation is that of $r+1$ forms. This is the focus of this paper. As a main tool, we first use the inverse system dictionary to relate an ideal $I \subset k\left[x_{1}, \ldots, x_{r}\right]$ generated by powers of linear forms to an ideal of fat points in $\mathbb{P}^{r-1}$, and then we show that the WLP problem of an ideal generated by powers of linear forms is closely connected to the geometry of the linear system of hypersurfaces in $\mathbb{P}^{r-2}$ of fixed degree with preassigned multiple points.

Let us briefly explain how this paper is organized. In Section 2 we give the connection between the WLP problem and Fröberg's conjecture. As a consequence, the results of this paper (about the failure of WLP for explicit ideals generated by $r+1$ powers of general linear forms in $r$ variables) can be interpreted as explicit results about when an ideal generated by $r+1$ powers of general linear forms in $r-1$ variables fails to have the Hilbert function predicted by Fröberg.

We begin Section 3 by explaining the tools that are applied throughout the paper. First, we recall a result of Emsalem and Iarrobino [1995] which gives a duality between powers of linear forms and ideals of fat points in $\mathbb{P}^{r-1}$. Then, we reduce our WLP problem to one of computing the Hilbert function of $n$ general fat points in $\mathbb{P}^{r-2}$ or, equivalently, to computing the dimension of the linear system of hypersurfaces in $\mathbb{P}^{r-2}$ of degree $d$ having some points of fixed multiplicity. Moreover, using Cremona transformations, one can relate two different linear systems to reduce the problem; see [Laface and Ugaglia 2006] or [Dumnicki 2009, Theorem 3].

In Section 4, we consider the case of 4 variables and we give a fairly complete answer about the failure of the WLP for

$$
I=\left\langle L_{1}^{a_{1}}, L_{2}^{a_{2}}, L_{3}^{a_{3}}, L_{4}^{a_{4}}, L_{5}^{a_{5}}\right\rangle \subset k\left[x_{1}, x_{2}, x_{3}, x_{4}\right],
$$

where the $L_{i}$ are general linear forms and $2 \leq a_{1} \leq a_{2} \leq a_{3} \leq a_{4} \leq a_{5}$. In Section 5, we deal with 5 variables and we completely determine when an ideal generated by uniform or almost uniform powers of six linear forms fails the WLP. We add some examples to illustrate that our methods extend beyond the mentioned results. The main result of Section 6 is Theorem 6.1, where we give a complete answer to the uniform case when the number of variables is even; in particular, we solve [Harbourne et al. 2011, Conjecture 5.5.2] when the number of variables is even. The case of an odd number of variables is left as an open conjecture and we present some evidence for this conjecture, including a proof for the case of seven variables.

Finally, it is worthwhile to point out that the approach used in this work can be applied to many other situations, in particular when the generators do not all 
have the same degree, but the calculations quickly become overwhelming. The key steps of our approach involve identifying the consecutive degrees where the WLP fails, determining the appropriate inequality (or equality) in the dimensions, and then after making the translation to fat points, showing that the dimension of the corresponding linear system has the predicted failure. As we will see, all of these steps become very intricate.

\section{Lefschetz properties and Fröberg's conjecture}

This section discusses briefly the relations between Fröberg's conjecture and the Lefschetz properties. For all references to Fröberg's work, see [Fröberg 1985].

Let $R=k\left[x_{1}, \ldots, x_{r}\right]$ be a polynomial ring over a field $k$ with its standard grading. Assume that $k$ is infinite. Denote by $h_{A}$ the Hilbert function

$$
h_{A}(t)=\operatorname{dim}_{k}[A]_{t}
$$

of a standard graded $k$-algebra $A$. Fix a positive integer $s$, and fix positive integers $d_{1}, \ldots, d_{s}$. Consider the product $P=[R]_{d_{1}} \times \cdots \times[R]_{d_{s}}$, with its Zariski topology. Fröberg's idea is that there should be a dense open subset $U$ of $P$ such that for all points $\left(F_{1}, \ldots, F_{s}\right)$ of $U$, the coordinate rings of the ideals $\left\langle F_{1}, \ldots, F_{s}\right\rangle$ should have the same Hilbert function, which was precisely described by Fröberg; we call this the expected Hilbert function for the given values of $s, d_{1}, \ldots, d_{s}$. (In particular, $U$ will avoid any instances where $F_{i}=F_{j}$.) Throughout this paper, when we say that an ideal of general forms has some property $(*)$, either the values of $s, d_{1}, \ldots, d_{s}$ will be understood from the context, or the statement will be valid for any choices of $s, d_{1}, \ldots, d_{s}$, and we will mean that there is a Zariski-open subset $U$ as above whose points correspond to generators of an ideal satisfying $(*)$. Beginning in the next section, usually we will have $s=r+1$.

Fröberg's conjecture is equivalent to the following structural statement: If $I \subset R$ is an ideal generated by $s$ general forms of degrees $d_{1}, \ldots, d_{s}$, and $f \in R$ is another general form of degree $d$, then the multiplication map

$$
\times f:[R / I]_{i-d} \rightarrow[R / I]_{i}
$$

has maximal rank for every integer $i$; that is, it is injective or surjective. This conjecture is known if the number of variables is at most three, due to Anick [1986], and open if the number of variables is greater.

Recall that an artinian graded algebra $A=R / I$ has the weak Lefschetz property $(W L P)$ if there is a linear form $\ell$ such that the multiplication $\times \ell:[A]_{i-1} \rightarrow[A]_{i}$ has maximal rank for each integer $i$. Furthermore, $A$ has the strong Lefschetz property $(S L P)$ if for each integer $d \geq 1$ the multiplication

$$
\times \ell^{d}:[A]_{i-d} \rightarrow[A]_{i}
$$


has maximal rank for each $i$. It is known that the WLP does not imply the SLP.

Fröberg's conjecture implies, in particular, that all ideals generated by general forms have the WLP. In fact, these concepts are even more closely related, as the following result and its corollaries show.

Proposition 2.1. (a) If Fröberg's conjecture is true for all ideals generated by general forms in $r$ variables, then all ideals generated by general forms in $r+1$ variables have the WLP.

(b) Let $R=k\left[x_{1}, \ldots, x_{r+1}\right], \ell \in R$ be a general linear form, and $S=R /\langle\ell\rangle \cong$ $k\left[x_{1}, \ldots, x_{r}\right]$. Fix positive integers $s, d_{1}, \ldots, d_{s+1}$. Let $L_{1}, L_{2}, \ldots, L_{s+1} \in R$ be linear forms. Denote by ${ }^{-}$the restriction from $R$ to $S \cong R /\langle\ell\rangle$. Make the following assumptions:

(i) The ideal $I=\left\langle L_{1}^{d_{1}}, \ldots, L_{s}^{d_{s}}\right\rangle$ has the WLP.

(ii) The multiplication $\times \bar{L}_{s+1}^{d_{s+1}}:[S / \bar{I}]_{j-d_{s+1}} \rightarrow[S / \bar{I}]_{j}$ has maximal rank.

Then $R /\left\langle L_{1}^{d_{1}}, \ldots, L_{s+1}^{d_{s+1}}\right\rangle$ has the WLP.

Proof. As pointed out in the paragraph preceding [Migliore and Miró-Roig 2003, Proposition 4.3], the proof of [ibid., Proposition 4.3] implies Claim (a). Essentially, it also proves Claim (b). For the reader's convenience, we give the proof of (b) here.

Let $A=R / I$. Let $f=L_{s+1}^{d_{s+1}}$. We have to show that $A / f A \cong R /(I, f)$ has the WLP. To this end, for simplicity let $d=d_{s+1}$ and consider the following commutative diagram with exact rows and columns,

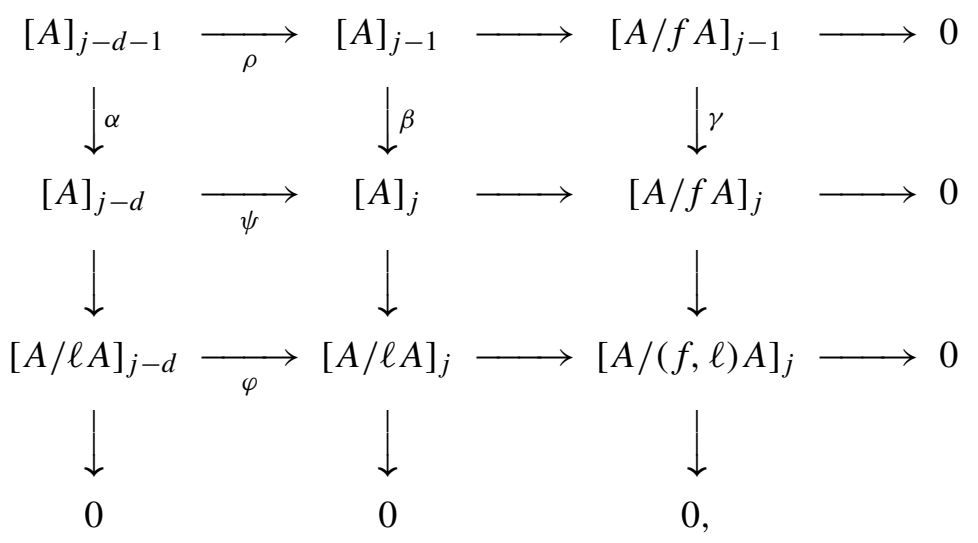

where $\alpha, \beta, \gamma$ are multiplications by $\ell$ and $\rho, \psi, \varphi$ are multiplications by $f$.

By (i), $\alpha$ and $\beta$ have maximal rank. We have to show that the same is true for $\gamma$. If $\beta$ is surjective, then so is $\gamma$. Thus, assume that $\beta$ is injective. Since the algebras in the bottom row are quotients of $\bar{R}=R / \ell R$ by ideals generated by powers of general linear forms in $\bar{R}$, the assumption implies that also $\varphi$ has maximal rank. If $\varphi$ is surjective, then so is $\gamma$, and we are done. If $\varphi$ is injective, then a routine diagram chase shows that $\gamma$ is injective as well, which completes the argument. 
The last result provides a new short proof of the main result of [Schenck and Seceleanu 2010].

Corollary 2.2 [Schenck and Seceleanu 2010]. If $k$ has characteristic zero, then every ideal generated by powers of linear forms in 3 variables has the WLP.

Proof. Let $J \subset R=k[x, y]$ be an ideal generated by powers of linear forms in two variables. By [Harima et al. 2003, Proposition 4.4], every ideal in two variables over a field of characteristic zero has the SLP. Hence, if $\ell$ in $R$ is a general linear form, then multiplication by any power of $\ell$, say $\ell^{e}$, on $R / J$ has maximal rank. However, since the statement is about ideals generated by powers of arbitrary linear forms, we need to show that the multiplication still has maximal rank whenever $\ell$ is not one of the linear forms whose powers generate $J$. To this end, it is enough to argue that the Hilbert function of the cokernel of multiplication by $\ell^{e}$, namely $R /\left(J, \ell^{e}\right)$, does not change. However, this is clear because the latter is determined by the Hilbert function of fat points in projective 1-space (see Theorem 3.3 below), which only depends on the degree of the scheme since it is defined by a principal ideal.

Now we can employ the argument used in the proof of Proposition 2.1. Indeed, we just showed that the map $\varphi$ in the commutative diagram above has maximal rank.

Corollary 2.3. Assume the characteristic is zero. Let $R=k\left[x_{1}, \ldots, x_{r+1}\right], \ell \in R$ be a general linear form, and $S=R /\langle\ell\rangle \cong k\left[x_{1}, \ldots, x_{r}\right]$. For integers $d_{1}, \ldots, d_{r+2}$, if an ideal of powers of general linear forms $\left\langle L_{1}^{d_{1}}, \ldots, L_{r+2}^{d_{r+2}}\right\rangle \subset R$ fails to have the $W L P$, then an ideal of powers of general linear forms $\left\langle\bar{L}_{1}^{d_{1}}, \ldots, \bar{L}_{r+2}^{d_{r+2}}\right\rangle \subset S$ fails to have the Hilbert function predicted by Fröberg's conjecture.

Proof. Taking $s=r+1$, condition (i) of Proposition 2.1(b) is satisfied by the result of [Stanley 1980; Watanabe 1987; Reid et al. 1991]. Thus (ii) must fail. We also know that $[S / \bar{I}]_{j-d_{r+1}}$ and $[S / \bar{I}]_{j}$ have the expected dimensions. The failure of $\times \bar{L}_{r+2}^{d_{r+2}}$ to have maximal rank then immediately gives the result.

If all ideals in $R=k\left[x_{1}, \ldots, x_{r}\right]$ that are generated by powers of general linear forms were to have the SLP, then these ideals would have the expected Hilbert function of ideals generated by general forms of the corresponding degrees, and thus Fröberg's conjecture would be true in $R$. Since complete intersections generated by powers of linear forms have the SLP, it follows that ideals generated by $r+1$ powers of general linear forms in $r$ variables have the expected Hilbert functions, so Fröberg's conjecture is true for ideals generated by $r+1$ general forms of $R$.

It has already been shown by Iarrobino in [1997] that ideals generated by $r+2$ powers of general linear forms in $r$ variables do not necessarily have the expected Hilbert function, leading to almost complete intersections generated by powers of 
general linear forms in $r+1$ variables that fail the WLP. By Corollary 2.2, this forces $r \geq 4$. Since complete intersections do have the WLP, these considerations suggest as a crucial test case the question of whether ideals generated by $r+1$ powers of general linear forms in $r \geq 4$ variables have the WLP.

In the following sections we study this phenomenon much more systematically. We give many explicit ideals of powers of $r+1$ general linear forms that fail the WLP, and so by Corollary 2.3 these all give examples in $r-1$ variables of ideals generated by powers of $r+1$ linear forms that fail to have the Hilbert function predicted by Fröberg. We will not repeat this remark after this section, but it is an important motivation for our work.

Example 2.4. For some specific examples, see [Migliore et al. 2011; Schenck and Seceleanu 2010]. However, we give two illustrations here.

(a) Taking $a_{1}=\cdots=a_{5}=5$, in Theorem 4.2 we have $\lambda=8$, so $k\left[x_{1}, \ldots, x_{4}\right] /$ $\left\langle x_{1}^{5}, \ldots, x_{4}^{5}, L^{5}\right\rangle$ fails the WLP from degree 7 to degree 8 . Thus the ideal generated by the fifth powers of five general linear forms in $k[x, y, z]$ fails to have the Hilbert function predicted by Fröberg.

(b) If $r$ is odd and $d>1$, then an ideal of powers of general linear forms of the form $\left\langle L_{1}^{d}, \ldots, L_{r}^{d}, L_{1}^{d}, L_{2}^{d}\right\rangle$ fails to have the Hilbert function predicted by Fröberg. This follows from Theorem 6.1 and the above discussion.

\section{General approach}

Let $R=k\left[x_{1}, \ldots, x_{r}\right]$ be a polynomial ring, where $k$ is a field of characteristic zero.

Notation 3.1. Throughout this paper, when $m$ is any integer, we will denote

$$
[m]_{+}=\max \{m, 0\} .
$$

For any artinian ideal $I \subset R$ and a general linear form $\ell \in R$, the exact sequence

$$
\cdots \rightarrow[R / I]_{m-1} \stackrel{\times \ell}{\longrightarrow}[R / I]_{m} \rightarrow[R /(I, \ell)]_{m} \rightarrow 0
$$

gives, in particular, that the multiplication by $\ell$ will fail to have maximal rank exactly when

$$
\operatorname{dim}_{k}[R /(I, \ell)]_{m} \neq \max \left\{\operatorname{dim}_{k}[R / I]_{m}-\operatorname{dim}_{k}[R / I]_{m-1}, 0\right\} ;
$$

in that case, we will say that $R / I$ fails the WLP in degree $m$.

Remark 3.2. Notice that to show that the multiplication by $\ell$ fails to have maximal rank from degree $m-1$ to degree $m$, it is enough to check the failure of injectivity if $\operatorname{dim}[R / I]_{m-1} \leq \operatorname{dim}[R / I]_{m}$, and it is enough to check the failure of surjectivity if $\operatorname{dim}[R / I]_{m-1} \geq \operatorname{dim}[R / I]_{m}$. We will then say that $R / I$ fails injectivity or fails 
surjectivity, respectively. An important part of the argument is the verification of the inequality of the dimensions.

In several of the papers mentioned above, this failure was studied via an examination of the splitting type of the first syzygy bundle of $I$. For powers of linear forms, we give an alternative approach, which we will implement in the subsequent sections.

We first recall a result of Emsalem and Iarrobino giving a duality between powers of linear forms and ideals of fat points in $\mathbb{P}^{n-1}$. We quote it in the form that we need.

Theorem 3.3 [Emsalem and Iarrobino 1995, Theorem I]. Let $\left\langle L_{1}^{a_{1}}, \ldots, L_{n}^{a_{n}}\right\rangle \subset R$ be an ideal generated by powers of $n$ linear forms. Let $\wp_{1}, \ldots, \wp_{n}$ be the ideals of $n$ points in $\mathbb{P}^{r-1}$. (Each point is actually obtained explicitly from the corresponding linear form by duality.) Choose positive integers $a_{1}, \ldots, a_{n}$. Then, for any integer $j \geq \max \left\{a_{i}\right\}$,

$$
\operatorname{dim}_{k}\left[R /\left\langle L_{1}^{a_{1}}, \ldots, L_{n}^{a_{n}}\right\rangle\right]_{j}=\operatorname{dim}_{k}\left[\wp_{1}^{j-a_{1}+1} \cap \cdots \cap \wp_{n}^{j-a_{n}+1}\right]_{j} .
$$

Now, we observe that the ideal $(I, \ell)$ is also an ideal generated by powers of linear forms! We conclude that if $\wp$ is the ideal of the point dual to $\ell$ then

$$
\operatorname{dim}_{k}\left[R /\left\langle L_{1}^{a_{1}}, \ldots, L_{n}^{a_{n}}, \ell\right\rangle\right]_{j}=\operatorname{dim}_{k}\left[\wp_{1}^{j-a_{1}+1} \cap \cdots \cap \wp_{n}^{j-a_{n}+1} \cap \wp^{j}\right]_{j} .
$$

Consider the points $P_{1}, \ldots, P_{n}, P$ in $\mathbb{P}^{r-1}$ defined by the ideals $\wp_{1}, \ldots, \wp_{n}, \wp$, respectively. Let $\lambda_{i}$ be the line joining $P$ to $P_{i}$, and let $H=\mathbb{P}^{r-2}$ be a general hyperplane defined by a linear form $L_{H}$. Let $Q_{i}$ be the point of intersection of $\lambda_{i}$ with $H$. For any positive integer $m$, we will denote by $\lambda_{i}^{m}$ the curve with defining ideal $I_{\lambda_{i}}^{m}$, and notice that $\lambda_{i}^{m}$ is arithmetically Cohen-Macaulay. Thus the hyperplane section of $\lambda_{i}^{m}$ by $H$ has saturated ideal $\mathfrak{q}_{i}^{m}=\left(I_{\lambda_{i}}^{m}, L_{H}\right) /\left(L_{H}\right)$ in the coordinate ring $R /\left(L_{H}\right)$ of $H$, and $\mathfrak{q}_{i}^{m}$ defines a fat point $Q_{i}^{m}$ in $H=\mathbb{P}^{r-2}$. The curve

$$
Y=\lambda_{1}^{j-a_{1}+1} \cup \cdots \cup \lambda_{n}^{j-a_{n}+1}
$$

is the cone over $Q_{1}^{a_{1}} \cup \cdots \cup Q_{n}^{a_{n}}$, and thus is also arithmetically Cohen-Macaulay. Proposition 3.4. Let $\left\langle L_{1}^{a_{1}}, \ldots, L_{n}^{a_{n}}\right\rangle \subset R$ be an ideal generated by powers of $n$ linear forms, and let $\ell$ be a general linear form. For $j \geq \max \left\{a_{i}\right\}$, we have

$$
\begin{aligned}
\operatorname{dim}_{k}\left[R /\left\langle L_{1}^{a_{1}}, \ldots, L_{n}^{a_{n}}, \ell\right\rangle\right]_{j} & \\
& =\operatorname{dim}_{k}\left[\wp_{1}^{j-a_{1}+1} \cap \cdots \cap \wp_{n}^{j-a_{n}+1} \cap \wp^{j}\right]_{j} \quad\left(\text { in } k\left[x_{1}, \ldots, x_{r}\right]\right) \\
& =\operatorname{dim}_{k}\left[\mathfrak{q}_{1}^{j-a_{1}+1} \cap \cdots \cap \mathfrak{q}_{n}^{j-a_{n}+1}\right]_{j} \quad\left(\text { in } k\left[x_{1}, \ldots, x_{r-1}\right]\right) .
\end{aligned}
$$

Proof. The first equality is Theorem 3.3. Without loss of generality let $P=$ $[0, \ldots, 0,1]$, with defining ideal $\wp=\left\langle x_{1}, \ldots, x_{r-1}\right\rangle$, and assume that $H$ is defined 
by $x_{r}=0$. Any form $F \in\left[\wp_{1}^{j-a_{1}+1} \cap \cdots \cap \wp_{n}^{j-a_{n}+1} \cap \wp^{j}\right]_{j}$ involves only the variables $x_{1}, \ldots, x_{r-1}$ since it is in $\left[\wp^{j}\right]_{j}$. Thus $F \in\left[I_{Y}\right]_{j}$, so viewing $F$ in $k\left[x_{1}, \ldots, x_{r-1}\right]$, we see that $F$ vanishes on the hyperplane section of $Y$; that is,

$$
F \in\left[\mathfrak{q}_{1}^{j-a_{1}+1} \cap \cdots \cap \mathfrak{q}_{n}^{j-a_{n}+1}\right]_{j}
$$

Now suppose $F$ satisfies $(*)$. Viewing $F$ in $k\left[x_{1}, \ldots, x_{r}\right]$ we see that $F$ vanishes on $Y$, hence also on the subscheme of $Y$ defined by $\wp_{1}^{j-a_{1}+1} \cap \cdots \cap \wp_{n}^{j-a_{n}+1}$. But we also have that $F \in \wp^{j}$, since it involves only $x_{1}, \ldots, x_{r-1}$. Moreover $F$ has degree $j$. Thus $\left.F \in \wp_{1}^{j-a_{1}+1} \cap \cdots \cap \wp_{n}^{j-a_{n}+1} \cap \wp^{j}\right]_{j}$ as desired.

Using Proposition 3.4, we reduce our WLP problem to one of computing the Hilbert function of $n$ general fat points in $\mathbb{P}^{r-2}$. From now on, we will denote by

$$
\mathscr{L}_{r-2}\left(j ; j-a_{1}+1, j-a_{2}+1, \ldots, j-a_{n}+1\right)
$$

the linear system $\left[\mathfrak{q}_{1}^{j-a_{1}+1} \cap \cdots \cap \mathfrak{q}_{n}^{j-a_{n}+1}\right]_{j} \subset\left[k\left[x_{1}, \ldots, x_{r-1}\right]\right]_{j}$. In order to simplify notation, we use superscripts to indicate repeated entries. For example, $\mathscr{L}_{3}\left(j ; 5^{2}, 2^{3}\right)=\mathscr{L}_{3}(j ; 5,5,2,2,2)$.

Notice that, for every linear system $\mathscr{L}_{r}\left(j ; a_{1}, \ldots, a_{n}\right)$, one has

$$
\operatorname{dim}_{k} \mathscr{L}_{r}\left(j ; a_{1}, \ldots, a_{n}\right) \geq \max \left\{0,\left(\begin{array}{c}
j+r \\
r
\end{array}\right)-\sum_{i=1}^{n}\left(\begin{array}{c}
a_{i}+r-1 \\
r
\end{array}\right)\right\},
$$

where the right-hand side is called the expected dimension of the linear system. If the inequality is strict, then the linear system $\mathscr{L}_{r}\left(j ; a_{1}, \ldots, a_{n}\right)$ is called special. It is a difficult problem to classify the special linear systems.

Using Cremona transformations, one can relate two different linear systems; see [Nagata 1960; Laface and Ugaglia 2006; Dumnicki 2009, Theorem 3].

Lemma 3.5. Let $n>r \geq 2$ and $j, a_{1}, \ldots, a_{n}$ be nonnegative integers, and set $m=(r-1) j-\left(a_{1}+\cdots+a_{r+1}\right)$. If $a_{i}+m \geq 0$ for all $i=1, \ldots, r+1$, then $\operatorname{dim}_{k} \mathscr{L}_{r}\left(j ; a_{1}, \ldots, a_{n}\right)=\operatorname{dim}_{k} \mathscr{L}_{r}\left(j+m ; a_{1}+m, \ldots, a_{r+1}+m, a_{r+2}, \ldots, a_{n}\right)$

Following [De Volder and Laface 2007], the linear system $\mathscr{L}_{r}\left(j ; a_{1}, \ldots, a_{n}\right)$ is said to be in standard form if $(r-1) j \geq a_{1}+\cdots+a_{r+1}$ and $a_{1} \geq \cdots \geq a_{n} \geq 0$. If $r=2$, then every linear system in standard form is nonspecial. This is no longer true if $r \geq 3$. However, De Volder and Laface were able to compute the speciality in the case of at most 8 fat points in $\mathbb{P}^{3}$. We state their result only in the form we need it. 
Theorem 3.6 [De Volder and Laface 2007, Theorem 5.3]. If the linear system $\mathscr{L}_{3}\left(j ; a_{1}, \ldots, a_{6}\right)$ is in standard form, then

$$
\operatorname{dim}_{k} \mathscr{L}_{3}\left(j ; a_{1}, \ldots, a_{6}\right)=\max \left\{0,\left(\begin{array}{c}
j+r \\
r
\end{array}\right)-\sum_{i=1}^{6}\left(\begin{array}{c}
a_{i}+r-1 \\
r
\end{array}\right)\right\}+\sum_{i=2}^{6}\left(\begin{array}{c}
t_{i}+1 \\
3
\end{array}\right)
$$

where $t_{i}=a_{1}+a_{i}-j$.

Notice that we always use the vector space dimension of the linear system rather than the dimension of its projectivization and that we adjusted the formula for the expected dimension. Furthermore, we always use the convention that a binomial coefficient $\left(\begin{array}{l}a \\ r\end{array}\right)$ is zero if $a<r$.

In this note, we are interested in certain almost complete intersections. Then one can compute the right-hand side of the inequality (3-1).

Lemma 3.7. Let $I=\left\langle L_{1}^{a_{1}}, \ldots, L_{r+1}^{a_{r+1}}\right\rangle \subset R$ be an almost complete intersection generated by powers of $r+1$ general linear forms. Then, for each integer $j$,

$$
\begin{aligned}
\operatorname{dim}_{k}[R / I]_{j}-\operatorname{dim}_{k}[R / I]_{j-1} & \\
= & {\left[h_{A}(j)-h_{A}\left(j-a_{r+1}\right)\right]_{+}-\left[h_{A}(j-1)-h_{A}\left(j-1-a_{r+1}\right)\right]_{+}, }
\end{aligned}
$$

where $A=R /\left\langle L_{1}^{a_{1}}, \ldots, L_{r}^{a_{r}}\right\rangle$. Furthermore, if $j \leq \frac{1}{2} a_{r+1}+\frac{1}{2} \sum_{i=1}^{r}\left(a_{i}-1\right)$, then the formula simplifies to

$$
\begin{aligned}
\operatorname{dim}_{k}[R / I]_{j}-\operatorname{dim}_{k}[R / I]_{j-1} & \\
= & {\left[h_{A}(j)-h_{A}(j-1)\right]-\left[h_{A}\left(j-a_{r+1}\right)-h_{A}\left(j-1-a_{r+1}\right)\right] . }
\end{aligned}
$$

Proof. Considering multiplication by $l_{r+1}^{a_{r+1}}$ on $A$, the first equation follows because the complete intersection $A$ has the SLP according to [Stanley 1980; Watanabe 1987; Reid et al. 1991]. The latter also implies that the Hilbert function of $A$ is unimodal. Its midpoint is $\frac{1}{2} \sum_{i=1}^{r}\left(a_{i}-1\right)$. Thus, the differences in brackets in the first formula are not negative if $j \leq \frac{1}{2} a_{r+1}+\frac{1}{2} \sum_{i=1}^{r}\left(a_{i}-1\right)$, proving the second formula.

Notice that the Hilbert function of the complete intersection $A$ can be computed using the Koszul complex that provides its minimal free resolution.

\section{Powers of linear forms in four variables}

In this section we let $R=k\left[x_{1}, x_{2}, x_{3}, x_{4}\right]$, where $k$ is a field of characteristic zero. Our main result will be to determine, in almost all cases, when an ideal generated by powers of five general linear forms has the WLP. To this end, without loss of generality we set $I=\left\langle x_{1}^{a_{1}}, x_{2}^{a_{2}}, x_{3}^{a_{3}}, x_{4}^{a_{4}}, L^{a_{5}}\right\rangle$, where $L$ is a general linear form and $a_{1} \leq a_{2} \leq a_{3} \leq a_{4} \leq a_{5}$. Part of the argument involves an application of Bézout's 
theorem to remove one-dimensional components of the relevant linear systems; this approach was also used in [Chandler 2005].

Lemma 4.1. Let $P_{1}, \ldots, P_{6}$, be points in $\mathbb{P}^{2}$ in linear general position. Assign multiplicities $m_{1}, \ldots, m_{6}$ respectively to the points, with $0 \leq m_{1} \leq \cdots \leq m_{6}$. (In particular, taking some of the $m_{i}=0$ allows us to consider fewer than six points.) Assume that $d \geq m_{5}+m_{6}$ and that $2 d \geq \sum_{i=2}^{6} m_{i}$. Then the fat point scheme $Z=m_{1} P_{1}+\cdots+m_{6} P_{6}$ imposes independent conditions on curves of degree $d$.

Proof. Let $X$ be the rational surface obtained by blowing up $\mathbb{P}^{2}$ at the points $P_{i}$. Let $L, E_{1}, \ldots, E_{6}$ be the standard basis of the divisor class group of $X$, that is, $L$ is the pullback of the class of a line in $\mathbb{P}^{2}$ and $E_{1}, \ldots, E_{6}$ are the exceptional divisors. Under the stated assumptions $d \geq m_{5}+m_{6}$ and $2 d \geq \sum_{i=2}^{6} m_{i}$, the divisor $d L-m_{1} E_{1}-\cdots-m_{6} E_{6}$ is numerically effective (nef), by [Di Rocco 1996, Theorem 3.4]. Then [Geramita et al. 2009, Theorem 2.3 and Remark 2.4] show that the Castelnuovo-Mumford regularity of $I_{Z}$ is $\leq d+1$. This implies the claimed result.

The following theorem represents "half" of our results (the "even" case) concerning when an ideal of five powers of general linear forms has the WLP. It is not a complete list, however, because of the second condition in (iii). See Remark 4.4 for further discussion.

Theorem 4.2. Let $L$ be a general linear form and let $I=\left\langle x_{1}^{a_{1}}, x_{2}^{a_{2}}, x_{3}^{a_{3}}, x_{4}^{a_{4}}, L^{a_{5}}\right\rangle$. Assume that $a_{1}+a_{2}+a_{3}+a_{4}$ is even. Let $\lambda=\left(a_{1}+a_{2}+a_{3}+a_{4}\right) / 2-2$.

(i) If $a_{5} \geq \lambda$ then the ring $R / I$ has the $W L P$.

(ii) If $a_{5}<\lambda$ and $a_{1}+a_{4} \geq a_{2}+a_{3}$ then $R / I$ fails the WLP from degree $\lambda-1$ to degree $\lambda$.

(iii) If $a_{5}<\lambda, a_{1}+a_{4}<a_{2}+a_{3}$ and $2 a_{5}+a_{1}-a_{2}-a_{3}-a_{4} \geq 0$ then $R / I$ fails the WLP from degree $\lambda-1$ to degree $\lambda$.

The hypotheses of (ii) and of (iii) both force $a_{1} \geq 3$.

Proof. Let $\ell$ be a general linear form. Letting $J=\left\langle x_{1}^{a_{1}}, x_{2}^{a_{2}}, x_{3}^{a_{3}}, x_{4}^{a_{4}}\right\rangle$, consider the commutative diagram

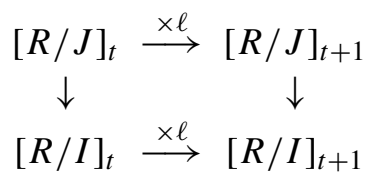

(where the vertical arrows are the natural restriction). If we know that the multiplication on the top row is surjective, then we immediately conclude surjectivity on the bottom row. Notice that $2 \lambda$ is the socle degree of the artinian complete intersection $R /\left(x_{1}^{a_{1}}, x_{2}^{a_{2}}, x_{3}^{a_{3}}, x_{4}^{a_{4}}\right)$, so $\lambda$ is the midpoint. 
First assume that $a_{5}>\lambda$. Then clearly $R / I$ and $R / J$ coincide in degrees $\leq \lambda$, so we have injectivity (by the result of Stanley, Watanabe, Reid, Roberts and Roitman) for $(\times \ell):[R / I]_{t} \rightarrow[R / I]_{t+1}$ for all $t \leq \lambda-1$. When $t \geq \lambda$ we have surjectivity for $R / J$, so by the above result we also have it for $R / I$.

Now assume that $a_{5}=\lambda$. We wish to show that $R / I$ has the WLP. Again surjectivity is immediate for $t \geq \lambda$, and injectivity is immediate for $t \leq \lambda-2$. When $t=\lambda-1$ we consider two cases. If $\operatorname{dim}[R / J]_{\lambda-1}=\operatorname{dim}[R / J]_{\lambda}$ then by StanleyWatanabe we have surjectivity for $(\times \ell):[R / J]_{\lambda-1} \rightarrow[R / J]_{\lambda}$, so the same holds for $R / I$. If $\operatorname{dim}[R / J]_{\lambda-1}<\operatorname{dim}[R / J]_{\lambda}$, the image of $[R / J]_{\lambda-1}$ in $[R / J]_{\lambda}$ under multiplication by $\ell$ is not surjective. Hence the vector space $\left[\left\langle x_{1}^{a_{1}}, x_{2}^{a_{2}}, x_{3}^{a_{3}}, x_{4}^{a_{4}}, \ell\right\rangle\right]_{\lambda}$ is not all of $R_{\lambda}$. But in characteristic zero the $\lambda$-th powers of linear forms span $[R]_{\lambda}$. Thus for a general $L$, the image of $L^{\lambda}$ in $[R / J]_{\lambda}$ is outside the image of $[R / J]_{\lambda-1}$ in $[R / J]_{\lambda}$. Thus $(\times \ell):[R / I]_{\lambda-1} \rightarrow[R / I]_{\lambda}$ is injective since it is for $R / J$ in that degree. This completes the proof of (i).

We now prove (ii). Our assumptions now are that $a_{1}+a_{2}+a_{3}+a_{4}$ is even, $a_{5}<\lambda$, and $a_{1}+a_{4} \geq a_{2}+a_{3}$. We first note that the hypotheses force $a_{1} \geq 3$, since if $a_{1}=2$ then $a_{5}<\lambda=\left(a_{2}+a_{3}+a_{4}-2\right) / 2 \leq\left(2+2 a_{4}-2\right) / 2=a_{4}$.

We will show that the multiplication by $\ell$ fails to have maximal rank from degree $\lambda-1$ to degree $\lambda$. If $\operatorname{dim}[R / I]_{\lambda-1} \leq \operatorname{dim}[R / I]_{\lambda}$ it is enough to check the failure of injectivity, and if $\operatorname{dim}[R / I]_{\lambda-1} \geq \operatorname{dim}[R / I]_{\lambda}$ it is enough to check the failure of surjectivity. (See Example 4.3.)

According to (3-1), the task is to show that

$$
\operatorname{dim}[R /(I, \ell)]_{\lambda}>\left[\operatorname{dim}[R / I]_{\lambda}-\operatorname{dim}[R / I]_{\lambda-1}\right]_{+} .
$$

We will compute the left-hand side using Proposition 3.4 and the right-hand side using Lemma 3.7.

By Proposition 3.4 and Theorem 3.3, we have $\operatorname{dim}_{k}[R /(I, \ell)]_{\lambda}$

$$
\begin{aligned}
& =\operatorname{dim}_{k}\left[\mathfrak{q}_{1}^{\lambda-a_{1}+1} \cap \mathfrak{q}_{2}^{\lambda-a_{2}+1} \cap \mathfrak{q}_{3}^{\lambda-a_{3}+1} \cap \mathfrak{q}_{4}^{\lambda-a_{4}+1} \cap \mathfrak{q}_{5}^{\lambda-a_{5}+1}\right] \lambda \\
& =\operatorname{dim}_{k} \mathscr{L}_{2}\left(\lambda ; \lambda-a_{1}+1, \lambda-a_{2}+1, \lambda-a_{3}+1, \lambda-a_{4}+1, \lambda-a_{5}+1\right) .
\end{aligned}
$$

Notice that $\lambda-a_{1}+1 \geq \cdots \geq \lambda-a_{5}+1 \geq 2$. The vector space defines a linear system, and we want to find its dimension. The first step is to understand the one-dimensional base locus, which has components of degree 2 and of degree 1 . We will use Bézout's theorem to formally reduce the degree of the polynomials and the order of vanishing at the points, without changing the dimension of the linear system. If the result has dimension zero then (4-2) is also zero.

Let $F_{\lambda} \in \mathscr{L}_{2}\left(\lambda ; \lambda-a_{1}+1, \lambda-a_{2}+1, \lambda-a_{3}+1, \lambda-a_{4}+1, \lambda-a_{5}+1\right)$. There is a unique quadratic polynomial $F_{2}$ vanishing at the five general points. By Bézout's 
theorem, if $F_{2}$ is not a factor of $F_{\lambda}$ then it intersects $F_{\lambda}$ with multiplicity $2 \lambda$. On the other hand, considering the multiplicities at the five points, we get that the curves meet with multiplicity at least $5 \lambda-\sum_{i=1}^{5} a_{i}+5$. But

$$
5 \lambda-\sum_{i=1}^{5} a_{i}+5 \leq 2 \lambda \Longleftrightarrow a_{5} \geq \frac{a_{1}+a_{2}+a_{3}+a_{4}}{2}-1=\lambda+1,
$$

a contradiction. Hence $F_{2}$ is a factor of $F_{\lambda}$. We next want to know what power of $F_{2}$ divides $F_{\lambda}$. Thus we want to know the smallest $i$ for which

$$
2(\lambda-2 i) \geq\left(\lambda-a_{1}+1-i\right)+\cdots+\left(\lambda-a_{5}+1-i\right),
$$

and this is equivalent to $i \geq \lambda-a_{5}+1$. Thus we have $F_{\lambda}=F_{2}^{\lambda-a_{5}+1} \cdot F_{2 a_{5}-\lambda-2}$, where $F_{2 a_{5}-\lambda-2} \in \mathscr{L}_{2}\left(2 a_{5}-\lambda-2 ; a_{5}-a_{1}, a_{5}-a_{2}, a_{5}-a_{3}, a_{5}-a_{4}\right)$. (Notice that now there are only four points, and some of these multiplicities might even be zero.)

Now we consider linear factors coming from the lines joining two of these four points. There are six such lines; we denote by $L_{i j}, 1 \leq i<j \leq 4$, the line (as well as the linear form) passing through the points with multiplicities $a_{5}-a_{i}$ and $a_{5}-a_{j}$. Notice that if $a_{5}-a_{i}>2 a_{5}-\lambda-2$ then there are no such forms $F_{2 a_{5}-\lambda-2}$, so the desired dimension is zero.

Arguing in a similar manner as above, we obtain that if $\left(a_{5}-a_{i}\right)+\left(a_{5}-a_{j}\right)>$ $2 a_{5}-\lambda-2$, then $L_{i j}$ appears as a factor

$$
\left(a_{5}-a_{i}\right)+\left(a_{5}-a_{j}\right)-\left(2 a_{5}-\lambda-2\right)=\lambda+2-a_{i}-a_{j}
$$

times. Thus, letting $A_{i j}=\left[\lambda+2-a_{i}-a_{j}\right]_{+}=\max \left\{\lambda+2-a_{i}-a_{j}, 0\right\}$, we see that (formally)

$$
F_{\lambda}=F_{2}^{\lambda-a_{5}+1} \cdot \prod_{1 \leq i<j \leq 4} L_{i j}^{A_{i j}} \cdot G, \quad \text { where } \operatorname{deg} G=2 a_{5}-\lambda-2-\sum_{1 \leq i<j \leq 4} A_{i j} .
$$

Notice that

$$
\text { if } 2 a_{5}-\lambda-2<\sum_{1 \leq i<j \leq 4} A_{i j} \text {, then } \operatorname{dim}\left[\left\langle x_{1}^{a_{1}}, x_{2}^{a_{2}}, x_{3}^{a_{3}}, x_{4}^{a_{4}}, L^{a_{5}}, \ell\right\rangle\right]_{\lambda}=0 .
$$

Now, since we have assumed $a_{1}+a_{4} \geq a_{2}+a_{3}$, observe that

$$
\begin{array}{lll}
a_{1}+a_{4}-a_{2}-a_{3} \geq 0 & a_{2}+a_{3}-a_{1}-a_{4} \leq 0 & a_{2}+a_{4}-a_{1}-a_{3} \geq 0 \\
a_{1}+a_{3}-a_{2}-a_{4} \leq 0 & a_{3}+a_{4}-a_{1}-a_{2} \geq 0 & a_{1}+a_{2}-a_{3}-a_{4} \leq 0 .
\end{array}
$$

We claim that after removing the one-dimensional base locus, we obtain a set of at most 4 fat points of uniform multiplicities (possibly 0 ). We have already seen that after removing the powers of $F_{2}$ we are left with the problem of finding the 
dimension of the forms of degree $2 a_{5}-\lambda-2$ passing through four general fat points with multiplicity $a_{5}-a_{1}, \ldots, a_{5}-a_{4}$. To compute this, we have to determine precisely what is left when we remove the lines. At each of the four points we compute the multiplicity of the fat point that remains after we remove the (multiple) lines passing through it in the base locus (which do not contribute to the desired dimension):

$$
\begin{aligned}
a_{5} & -a_{4}-\sum_{i=1,2,3}\left[\lambda+2-a_{i}-a_{4}\right]_{+} \\
& =a_{5}-a_{4}-\left[\frac{-a_{1}+a_{2}+a_{3}-a_{4}}{2}\right]_{+}-\left[\frac{a_{1}-a_{2}+a_{3}-a_{4}}{2}\right]_{+}-\left[\frac{a_{1}+a_{2}-a_{3}-a_{4}}{2}\right]_{+} \\
& =a_{5}-a_{4} .
\end{aligned}
$$

Similarly,

$$
\begin{aligned}
& a_{5}-a_{3}-\sum_{i=1,2,4}\left[\lambda+2-a_{i}-a_{3}\right]_{+}=a_{5}-a_{4}, \\
& a_{5}-a_{2}-\sum_{i=1,3,4}\left[\lambda+2-a_{i}-a_{2}\right]_{+}=a_{5}-a_{4}, \\
& a_{5}-a_{1}-\sum_{i=2,3,4}\left[\lambda+2-a_{i}-a_{1}\right]_{+}=a_{5}-a_{4} .
\end{aligned}
$$

Concluding, we want to find the dimension of the vector space of forms of degree

$$
2 a_{5}-\lambda-2-\sum_{1 \leq i<j \leq 4} A_{i j}=2\left(a_{5}-a_{4}\right)
$$

passing through four points with multiplicity $a_{5}-a_{4}$. By Lemma 4.1, this dimension is $a_{5}-a_{4}+1$ (which in particular is at least 1). That is,

$$
\operatorname{dim}\left[\left\langle x_{1}^{a_{1}}, x_{2}^{a_{2}}, x_{3}^{a_{3}}, x_{4}^{a_{4}}, L^{a_{5}}, \ell\right\rangle\right]_{\lambda}=a_{5}-a_{4}+1 \geq 1 .
$$

Using Lemma 3.7, we now compute the "expected" dimension, that is, the right-hand side of (4-1). Let $A=R / J$, where $J=\left\langle x_{1}^{a_{1}}, x_{2}^{a_{2}}, x_{3}^{a_{3}}, x_{4}^{a_{4}}\right\rangle$. In the following, recall also that $\lambda$ is the midpoint of the $h$-vector of $R / J$, so $0 \leq h_{R / I}(\lambda)=$ $h_{A}(\lambda)-h_{A}\left(\lambda-a_{5}\right)$. Observe that $a_{4}<a_{1}+a_{2}+a_{3}$ since otherwise

$$
a_{5}-\lambda \geq a_{4}-\lambda=\frac{a_{4}-a_{1}-a_{2}-a_{3}}{2}+2>0,
$$

contradicting our assumption that $a_{5}<\lambda$. This implies, in particular, that for determining $h_{A}(\lambda)$ by using the Koszul resolution of $A$, we only need to consider the free modules up to $\bigoplus_{1 \leq i<l \leq 4} R\left(-a_{i}-a_{j}\right)$.

From (4-5) we see that only $\lambda-a_{2}-a_{3}+2, \lambda-a_{1}-a_{3}+2$ and $\lambda-a_{1}-a_{2}+2$ can be positive. Similarly, we have $\lambda-a_{5}-a_{i}+2 \leq 0$ since $a_{1}+a_{4} \geq a_{2}+a_{3}$. 
Then the "expected dimension" is

$$
\begin{aligned}
{\left[\operatorname{dim}[R / I]_{\lambda}-\operatorname{dim}[R / I]_{\lambda-1}\right]_{+} } \\
\quad=\left[h_{A}(\lambda)-h_{A}\left(\lambda-a_{5}\right)-h_{A}(\lambda-1)+h_{A}\left(\lambda-a_{5}-1\right)\right]_{+} \\
\quad=\left[\left(\begin{array}{c}
\lambda+2 \\
2
\end{array}\right)-\sum_{i=1}^{4}\left(\begin{array}{c}
\lambda-a_{i}+2 \\
2
\end{array}\right)+\sum_{1 \leq i<j \leq 3}\left(\begin{array}{c}
\lambda-a_{i}-a_{j}+2 \\
2
\end{array}\right)-\left(\begin{array}{c}
\lambda-a_{5}+2 \\
2
\end{array}\right)\right]_{+}
\end{aligned}
$$

If this is zero then clearly the actual dimension exceeds the expected one and we are done. If not, one verifies (we used CoCoA version 4.7.5 [CoCoA 2009]) that $\operatorname{dim}_{k}[R /(I, \ell)]_{\lambda}-\left[\operatorname{dim}[R / I]_{\lambda}-\operatorname{dim}[R / I]_{\lambda-1}\right]_{+}$

$$
\begin{aligned}
= & \left(a_{5}-a_{4}+1\right) \\
& -\left(\left(\begin{array}{c}
\lambda+2 \\
2
\end{array}\right)-\sum_{i=1}^{4}\left(\begin{array}{c}
\lambda-a_{i}+2 \\
2
\end{array}\right)+\sum_{1 \leq i<j \leq 3}\left(\begin{array}{c}
\lambda-a_{i}-a_{j}+2 \\
2
\end{array}\right)-\left(\begin{array}{c}
\lambda-a_{5}+2 \\
2
\end{array}\right)\right) \\
= & \left(\begin{array}{c}
\lambda+1-a_{5} \\
2
\end{array}\right),
\end{aligned}
$$

and this last binomial coefficient is at least 1 . Thus, in either case we have shown the inequality (4-1), which completes the proof of (ii).

Finally we prove (iii). Our assumptions now are that $a_{1}+a_{2}+a_{3}+a_{4}$ is even, $a_{5}<\lambda, a_{1}+a_{4}<a_{2}+a_{3}$ and $2 a_{5}+a_{1}-a_{2}-a_{3}-a_{4} \geq 0$. The calculations from (ii) continue to be valid up to (4-3) and (4-4). We first note that the hypotheses again force $a_{1} \geq 3$ since if $a_{1}=2$ then $a_{5}<\lambda=\left(a_{2}+a_{3}+a_{4}-2\right) / 2 \leq\left(2+2 a_{5}-2\right) / 2=a_{5}$.

In our current situation, though, observe that

$$
\begin{array}{lll}
a_{3}+a_{4}-a_{1}-a_{2}>0 & a_{1}+a_{2}-a_{3}-a_{4}<0 & a_{2}+a_{4}-a_{1}-a_{3}>0 \\
a_{1}+a_{3}-a_{2}-a_{4}<0 & a_{2}+a_{3}-a_{1}-a_{4}>0 & a_{1}+a_{4}-a_{2}-a_{3}<0 .
\end{array}
$$

Now we examine the linear system remaining after we remove the one-dimensional base locus. As in (ii), we obtain a linear system of curves of degree

$$
2 a_{5}-\lambda-2-\sum_{1 \leq i<j \leq 4} A_{i j}
$$

Now we compute the order of vanishing at the four points:

$$
\begin{aligned}
a_{5} & -a_{4}-\sum_{i=1,2,3}\left[\lambda+2-a_{i}-a_{4}\right]_{+} \\
& =a_{5}-a_{4}-\left[\frac{-a_{1}+a_{2}+a_{3}-a_{4}}{2}\right]_{+}-\left[\frac{a_{1}-a_{2}+a_{3}-a_{4}}{2}\right]_{+}-\left[\frac{a_{1}+a_{2}-a_{3}-a_{4}}{2}\right]_{+} \\
& =a_{5}-\frac{-a_{1}+a_{2}+a_{3}+a_{4}}{2} .
\end{aligned}
$$


By the additional hypothesis $2 a_{5}+a_{1}-a_{2}-a_{3}-a_{4} \geq 0$, this order of vanishing is $\geq 0$. A similar computation gives that the order of vanishing at the other three points is the same. Thus Lemma 4.1 shows that

$$
\operatorname{dim}\left[\left\langle x_{1}^{a_{1}}, x_{2}^{a_{2}}, x_{3}^{a_{3}}, x_{4}^{a_{4}}, L^{a_{5}}, \ell\right\rangle\right]_{\lambda}=a_{5}-\frac{-a_{1}+a_{2}+a_{3}+a_{4}}{2}+1 \geq 1 .
$$

The computation of the "expected" dimension is very similar to what we did above. From (4-7) we see that only $\lambda-a_{1}-a_{2}+2, \lambda-a_{1}-a_{3}+2$ and $\lambda-a_{1}-a_{4}+2$ can be positive. We again have $a_{4}<a_{1}+a_{2}+a_{3}$. Thus, Lemma 3.7 provides

$$
\begin{aligned}
{\left[\operatorname{dim}[R / I]_{\lambda}-\operatorname{dim}[R / I]_{\lambda-1}\right]_{+} } \\
=\left[h_{A}(\lambda)-h_{A}\left(\lambda-a_{5}\right)-h_{A}(\lambda-1)+h_{A}\left(\lambda-a_{5}-1\right)\right]_{+} \\
\quad=\left[\left(\begin{array}{c}
\lambda+2 \\
2
\end{array}\right)-\sum_{i=1}^{4}\left(\begin{array}{c}
\lambda-a_{i}+2 \\
2
\end{array}\right)+\sum_{i=2}^{4}\left(\begin{array}{c}
\lambda-a_{1}-a_{i}+2 \\
2
\end{array}\right)-\left(\begin{array}{c}
\lambda-a_{5}+2 \\
2
\end{array}\right)\right]_{+}
\end{aligned}
$$

As above, if this is zero then we are done. If not, one verifies (for example with CoCoA) that

$$
\begin{aligned}
& \operatorname{dim}_{k}[R /(I, \ell)]_{\lambda}-\left[\operatorname{dim}[R / I]_{\lambda}-\operatorname{dim}[R / I]_{\lambda-1}\right]_{+} \\
&= a_{5}-\frac{-a_{1}+a_{2}+a_{3}+a_{4}}{2}+1 \\
&-\left(\left(\begin{array}{c}
\lambda+2 \\
2
\end{array}\right)-\sum_{i=1}^{4}\left(\begin{array}{c}
\lambda-a_{i}+2 \\
2
\end{array}\right)+\sum_{i=2}^{4}\left(\begin{array}{c}
\lambda-a_{1}-a_{i}+2 \\
2
\end{array}\right)-\left(\begin{array}{c}
\lambda-a_{5}+2 \\
2
\end{array}\right)\right) \\
&=\left(\begin{array}{c}
\lambda+1-a_{5} \\
2
\end{array}\right),
\end{aligned}
$$

and this last binomial coefficient is at least 1 , establishing the inequality (4-1). This completes the proof of (iii).

Example 4.3. To illustrate that sometimes it is injectivity that fails and sometimes it is surjectivity, consider the following (produced using CoCoA).

When $a_{1}=5, a_{2}=7, a_{3}=8, a_{4}=10, a_{5}=10$ we get $\operatorname{dim}[R / I]_{12}=225$ and $\operatorname{dim}[R / I]_{13}=220$, so we expect surjectivity, but the image under multiplication by a general linear form has dimension 219.

When $a_{1}=5, a_{2}=7, a_{3}=8, a_{4}=10, a_{5}=12$ we get $\operatorname{dim}[R / I]_{12}=234$, $\operatorname{dim}[R / I]_{13}=236$, so we expect injectivity, but the image under multiplication by a general linear form has dimension 233.

Let us now discuss cases that are not covered by Theorem 4.2, still assuming that $a_{1}+a_{2}+a_{3}+a_{4}$ is even.

Remark 4.4. As above, let $I=\left\langle x_{1}^{a_{1}}, x_{2}^{a_{2}}, x_{3}^{a_{3}}, x_{4}^{a_{4}}, L^{a_{5}}\right\rangle$. Assume $2 a_{5}+a_{1}-a_{2}-$ $a_{3}-a_{4}<0$ and $a_{1} \geq 3$. Then: 
(i) If $a_{1}=2$ then $R / I$ has the WLP (see Theorem 4.6).

(ii) If $a_{1}=3$, then in the following cases, $R / I$ fails to have the WLP, and the failure is in degree $\lambda-1$ and it fails by 1 .

- $(3,9, m, m, m)$ for $m \geq 9$

- $(3,10, m, m+1, m+1)$ for $m \geq 10$

- $(3,11, m, m, m+1)$ for $m \geq 11$

- $(3,11, m, m+2, m+2)$ for $m \geq 11$

- $(3,12, m, m+1, m+2)$ for $m \geq 12$

- $(3,12, m, m+3, m+3)$ for $m \geq 12$

- $(3,13, m, m, m+2)$ for $m \geq 13$

- $(3,13, m, m+2, m+3)$ for $m \geq 13$

- $(3,13, m, m+4, m+4)$ for $m \geq 13$

(This is shown using arguments as in the proof of Theorem 4.2. We omit the details.)

Furthermore, the reader can easily construct examples using CoCoA to support the following statements, although we do not have proofs (see also the beginning of the proof of Theorem 6.5):

(iii) When $a_{1}=3$ and $a_{2} \leq 13$, all cases apart from the ones above have the WLP.

(iv) When $a_{1}=3,4$, some examples have the WLP and others do not.

(v) When $a_{1} \geq 5, R / I$ fails the WLP.

We now consider the case where $a_{1}+a_{2}+a_{3}+a_{4}$ is odd. There are some interesting differences to Theorem 4.2. As before, Theorem 4.5 is not a complete classification because of the extra condition in (iii).

Theorem 4.5. Let $L$ be a general linear form and let $I=\left\langle x_{1}^{a_{1}}, x_{2}^{a_{2}}, x_{3}^{a_{3}}, x_{4}^{a_{4}}, L^{a_{5}}\right\rangle$. Assume that $a_{1}+a_{2}+a_{3}+a_{4}$ is odd. Let $\lambda=\left(a_{1}+a_{2}+a_{3}+a_{4}-5\right) / 2$.

(i) If $a_{5} \geq \lambda-1$ then the ring $R / I$ has the WLP.

(ii) If $a_{5}<\lambda-1$ and $a_{1}+a_{4} \geq a_{2}+a_{3}$ then $R / I$ fails the WLP from degree $\lambda-1$ to degree $\lambda$. These hypotheses force $a_{1} \geq 5$.

(iii) If $a_{5}<\lambda-1, a_{1}+a_{4}<a_{2}+a_{3}$ and $2 a_{5}+3-a_{4}-a_{3}-a_{2}+a_{1} \geq 0$ then $R / I$ fails the WLP from degree $\lambda-1$ to degree $\lambda$. These hypotheses force $a_{1} \geq 3$.

Proof. The outline of part of the proof is the same as that for Theorem 4.2, and we only highlight the differences. First note that with the hypotheses of (ii), if $a_{1} \leq 4$ then we have $a_{5}<\lambda-1=\left(a_{1}+a_{2}+a_{3}+a_{4}-7\right) / 2 \leq\left(2 a_{1}+2 a_{4}-7\right) / 2 \leq a_{4}+(1) / 2$, a contradiction. Similarly, with the hypotheses of (iii), if $a_{1}=2$ then we have $a_{2}+a_{3}+a_{4}-5 \leq 2 a_{5}<2 \lambda-2=a_{2}+a_{3}+a_{4}-5$, again a contradiction. 
Now, for all three parts of the theorem, we show that the one-dimensional part of the base locus corresponding to the quadratic polynomial $F_{2}$ has equation $F_{2}^{\lambda-5}$. Thus, in the first step we want to compute

$$
\operatorname{dim} \mathscr{L}_{2}\left(2 a_{5}-\lambda ; a_{5}-a_{1}+1, a_{5}-a_{2}+1, a_{5}-a_{3}+1, a_{5}-a_{4}+1,1\right) .
$$

In the second step, we obtain that if $\left(a_{5}-a_{i}+1\right)+\left(a_{5}-a_{j}+1\right)>2 a_{5}-\lambda$ then $L_{i j}$ appears as a factor

$$
\left(a_{5}-a_{i}+1\right)+\left(a_{5}-a_{j}+1\right)-\left(2 a_{5}-\lambda\right)=\lambda-a_{i}-a_{j}+2
$$

times. Thus we let $A_{i j}=\left[\lambda+2-a_{i}-a_{j}\right]_{+}$as before, and formally we have

$$
F_{\lambda}=F_{2}^{\lambda-a_{5}} \cdot \prod_{1 \leq i<j \leq 4} L_{i j}^{A_{i j}} \cdot G, \quad \text { where } \quad \operatorname{deg} G=2 a_{5}-\lambda-\sum_{1 \leq i<j \leq 4} A_{i j} .
$$

Notice that

$$
\text { if } \quad 2 a_{5}-\lambda<\sum_{1 \leq i<j \leq 4} A_{i j} \quad \text { then } \operatorname{dim}\left[\left\langle x_{1}^{a_{1}}, x_{2}^{a_{2}}, x_{3}^{a_{3}}, x_{4}^{a_{4}}, L^{a_{5}}, \ell\right\rangle\right] \lambda=0 .
$$

For (i), we want to show that whenever $a_{5} \geq \lambda-1$, the multiplication $(\times \ell)$ : $[R / I]_{t} \rightarrow[R / I]_{t+1}$ has maximal rank. Let $J=\left\langle x_{1}^{a_{1}}, x_{2}^{a_{2}}, x_{3}^{a_{3}}, x_{4}^{a_{4}}\right\rangle$. Notice that we have $h_{R / J}(\lambda)=h_{R / J}(\lambda+1)$. We consider several cases.

- If $t \geq \lambda$, we know that the multiplication on $R / J$ from degree $t$ to degree $t+1$ is surjective, by the result of Watanabe, Stanley, Reid, Roberts and Roitman. Since $R / I$ is a quotient, the same holds for $R / I$. This holds no matter what $a_{5}$ is.

- If $t \leq a_{5}-2$, then $[R / I]_{t}=[R / J]_{t}$ and $[R / I]_{t+1}=[R / J]_{t+1}$, so again the result follows trivially. Notice that as a result of these first two cases, we are done if $a_{5} \geq \lambda+1$.

- If $\left(t, a_{5}\right)=(\lambda-1, \lambda)$ or $\left(t, a_{5}\right)=(\lambda-2, \lambda-1)$, we know that the multiplication for $R / J$ is injective in either of these cases, and that $\operatorname{dim}[R / I]_{t}=\operatorname{dim}[R / J]_{t}$ and $\operatorname{dim}[R / I]_{t+1}=\operatorname{dim}[R / J]_{t+1}-1$. Then we argue exactly as in the case $a_{5}=\lambda$ at the beginning of the proof of Theorem 4.2 , using that the $\lambda$-th and $(\lambda-1)$-st powers of linear forms span $[R]_{\lambda}$ and $[R]_{\lambda-1}$, respectively. In particular, this completes the argument if $a_{5}=\lambda$.

- The case where $t=\lambda-1, a_{5}=\lambda-1$ is the most subtle, and we now give the argument for this case.

We claim that the multiplication $(\times \ell):[R / I]_{\lambda-1} \rightarrow[R / I]_{\lambda}$ is injective. To see this, we will show that $\operatorname{dim}[R / I]_{\lambda-1}<\operatorname{dim}[R / I]_{\lambda}$ and that $\operatorname{dim}[R /(I, \ell)]_{\lambda}=$ $\operatorname{dim}[R / I]_{\lambda}-\operatorname{dim}[R / I]_{\lambda-1}$. 
First we compute $\operatorname{dim}[R /(I, \ell)]_{\lambda}$. The first step (4-8) now becomes

$$
\operatorname{dim}[R /(I, \ell)]_{\lambda}=\operatorname{dim} \mathscr{L}_{2}\left(\lambda-2 ; \lambda-a_{1}, \lambda-a_{2}, \lambda-a_{3}, \lambda-a_{4}, 1\right) .
$$

We now consider three cases. First, if $a_{1}=a_{2}=a_{3}=a_{4}-1$ then (4-8) becomes

$$
\begin{aligned}
\operatorname{dim}[R /(I, \ell)]_{\lambda} & =\operatorname{dim} \mathscr{L}_{2}\left(2 a_{4}-6 ; a_{4}-3, a_{4}-3, a_{4}-3, a_{4}-4,1\right) \\
& =2 a_{4}-6=2 a_{1}-4
\end{aligned}
$$

thanks to Lemma 4.1.

We now assume that we are not in the first case. We obtain

$$
\begin{array}{ll}
A_{1,2}=\left[\frac{-a_{1}-a_{2}+a_{3}+a_{4}-1}{2}\right]_{+} \geq 0 & A_{1,3}=\left[\frac{-a_{1}+a_{2}-a_{3}+a_{4}-1}{2}\right]_{+} \geq 0 \\
A_{1,4}=\left[\frac{-a_{1}+a_{2}+a_{3}-a_{4}-1}{2}\right]_{+} & A_{2,3}=\left[\frac{a_{1}-a_{2}-a_{2}+a_{4}-1}{2}\right]_{+} \\
A_{2,4}=\left[\frac{a_{1}-a_{2}+a_{3}-a_{4}-1}{2}\right]_{+}=0 & A_{3,4}=\left[\frac{a_{1}+a_{2}-a_{3}-a_{4}-1}{2}\right]_{+}=0 .
\end{array}
$$

Our second case is $a_{2}+a_{3}>a_{1}+a_{4}$. Then $A_{1,2}, A_{1,3}$ and $A_{1,4}$ are possibly nonzero. A calculation shows that we then must have

$$
\begin{aligned}
\operatorname{dim}[R /(I, \lambda)]_{\lambda} & =\operatorname{dim} \mathscr{L}_{2}\left(2 a_{1}-3 ; a_{1}-1, a_{1}-2, a_{1}-2, a_{1}-2,1\right) \\
& =2 a_{1}-3 .
\end{aligned}
$$

Our third case is $a_{1}+a_{4}>a_{2}+a_{3}$. Then $A_{1,2}, A_{1,3}$ and $A_{2,3}$ are possibly nonzero. Another calculation shows that we must have

$$
\begin{aligned}
\operatorname{dim}[R /(I, \ell)]_{\lambda} & =\operatorname{dim} \mathscr{L}_{2}\left(a_{1}+a_{2}+a_{3}-a_{4}-3 ;\left(\lambda-a_{4}+1\right)^{3}, \lambda-a_{4}, 1\right) \\
& =a_{1}+a_{2}+a_{3}-a_{4}-3 .
\end{aligned}
$$

Now we compute the expected dimension $\operatorname{dim}[R / I]_{\lambda}-\operatorname{dim}[R / I]_{\lambda-1}$ (recall $\left.a_{5}=\lambda-1\right)$ by using Lemma 3.7 .

In the first case $\left(a_{1}=a_{2}=a_{3}=a_{4}-1\right)$ we use the Koszul complex and easily compute that the expected dimension is $2 a_{4}-6=2 a_{1}-4$, agreeing with (4-11).

In the second case, using the observations about which $A_{i, j}$ are positive, the Koszul resolution gives the expected dimension $2 a_{1}-3$, agreeing with (4-12).

In the third case, again using the observations about the $A_{i, j}$ and the Koszul complex, we obtain the expected dimension $a_{1}+a_{2}+a_{3}-a_{4}-3$, agreeing with (4-13). Thus when $a_{5}=\lambda-1, R / I$ has the WLP, concluding the proof of (i).

Now, we prove (ii). Hence, we are assuming that $a_{1}+a_{4} \geq a_{2}+a_{3}$. In fact, since $a_{1}+a_{2}+a_{3}+a_{4}$ is odd, we actually have $a_{1}+a_{4}>a_{2}+a_{3}$. We obtain

$$
\begin{array}{lll}
a_{1}+a_{4}-a_{2}-a_{3}>0, & a_{2}+a_{3}-a_{1}-a_{4}<0, & a_{2}+a_{4}-a_{1}-a_{3}>0, \\
a_{1}+a_{3}-a_{2}-a_{4}<0, & a_{3}+a_{4}-a_{1}-a_{2}>0, & a_{1}+a_{2}-a_{3}-a_{4}<0 .
\end{array}
$$


Therefore, we get

$$
\begin{aligned}
a_{5}-a_{4}+1-\sum_{i=1,2,3}\left[\lambda+2-a_{i}-a_{4}\right]_{+} & =a_{5}-a_{4}+1, \\
a_{5}-a_{3}+1-\sum_{i=1,2,4}\left[\lambda+2-a_{i}-a_{3}\right]_{+} & =a_{5}-a_{4}+2, \\
a_{5}-a_{2}+1-\sum_{i=1,3,4}\left[\lambda+2-a_{i}-a_{2}\right]_{+} & =a_{5}-a_{4}+2, \\
a_{5}-a_{1}+1-\sum_{i=2,3,4}\left[\lambda+2-a_{i}-a_{1}\right]_{+} & =a_{5}-a_{4}+2, \\
2 a_{5}-\lambda-\sum_{1 \leq i<j \leq 4} A_{i j} & =2\left(a_{5}-a_{4}+2\right) .
\end{aligned}
$$

Concluding, we want the dimension of the linear system

$$
\mathscr{L}_{2}\left(2 a_{5}-2 a_{4}+4 ; a_{5}-a_{4}+2, a_{5}-a_{4}+2, a_{5}-a_{4}+2, a_{5}-a_{4}+1,1\right) .
$$

By Lemma 4.1 we have

$$
\begin{aligned}
\operatorname{dim}_{k} \mathscr{L}_{2}\left(2 a_{5}-2 a_{4}+4 ;\right. & \left.\left(a_{5}-a_{4}+2\right)^{3}, a_{5}-a_{4}+1,1\right) \\
\geq & \operatorname{dim}_{k} \mathscr{L}_{2}\left(2 a_{5}-2 a_{4}+4 ;\left(a_{5}-a_{4}+2\right)^{3}, a_{5}-a_{4}+1\right)-1 \\
& =\operatorname{dim}_{k} \mathscr{L}_{2}\left(2 a_{5}-2 a_{4}+4 ;\left(a_{5}-a_{4}+2\right)^{4}\right)+a_{5}-a_{4}+1 \\
& =2 a_{5}-2 a_{4}+4 .
\end{aligned}
$$

This is clearly positive. Arguing as in the proof of Theorem 4.2, and using the inequalities (4-14), we compute the expected dimension and we get

$\left[\operatorname{dim}[R / I]_{\lambda}-\operatorname{dim}[R / I]_{\lambda-1]}\right]_{+}$

$$
=\left[\left(\begin{array}{c}
\lambda+2 \\
2
\end{array}\right)-\sum_{i=1}^{4}\left(\begin{array}{c}
\lambda-a_{i}+2 \\
2
\end{array}\right)+\sum_{1 \leq i<j \leq 3}\left(\begin{array}{c}
\lambda-a_{j}-a_{i}+2 \\
2
\end{array}\right)-\left(\begin{array}{c}
\lambda-a_{5}+2 \\
2
\end{array}\right)\right]_{+} .
$$

If the part inside the brackets is negative, the actual dimension clearly exceeds the expected one, and we are done. If not, a straightforward computation shows that

$$
\begin{aligned}
& \operatorname{dim}_{k}[R /(I, \ell)]_{\lambda}-\left[\operatorname{dim}_{k}[R / I]_{\lambda}-\operatorname{dim}_{k}[R / I]_{\lambda-1}\right] \\
& \geq\left(2 a_{5}-2 a_{4}+4\right) \\
& \quad-\left(\left(\begin{array}{c}
\lambda+2 \\
2
\end{array}\right)-\sum_{i=1}^{4}\left(\begin{array}{c}
\lambda-a_{i}+2 \\
2
\end{array}\right)+\sum_{1 \leq i<j \leq 3}\left(\begin{array}{c}
\lambda-a_{j}-a_{i}+2 \\
2
\end{array}\right)-\left(\begin{array}{c}
\lambda-a_{5}+2 \\
2
\end{array}\right)\right) \\
& =\left(\begin{array}{c}
\lambda-a_{5} \\
2
\end{array}\right)>0 .
\end{aligned}
$$

Thus the actual dimension exceeds the expected dimension, and this completes the proof of (ii).

(iii) We break this into two cases: first we will assume that $a_{1}+a_{4}+3 \leq a_{2}+a_{3}$, and then we will handle the case $a_{1}+a_{4}+1=a_{2}+a_{3}$. 
So to begin, our assumptions now are that $a_{1}+a_{2}+a_{3}+a_{4}$ is odd, $a_{5}<\lambda-1$, $a_{1}+a_{4}+3 \leq a_{2}+a_{3}$ and $2 a_{5}+3-a_{4}-a_{3}-a_{2}+a_{1} \geq 0$. Hence, we have

$$
\begin{array}{lll}
a_{3}+a_{4}-a_{1}-a_{2}>0, & a_{1}+a_{2}-a_{3}-a_{4}<0, & a_{2}+a_{4}-a_{1}-a_{3}>0, \\
a_{1}+a_{3}-a_{2}-a_{4}<0, & a_{2}+a_{3}-a_{1}-a_{4}>0, & a_{1}+a_{4}-a_{2}-a_{3}<0 .
\end{array}
$$

Now we examine the linear system remaining after we remove the one-dimensional base locus. Observe that

$$
\begin{aligned}
& a_{5}-a_{4}+1-\sum_{i=1,2,3}\left[\lambda+2-a_{i}-a_{4}\right]_{+}=a_{5}+1-\left(a_{2}+a_{3}+a_{4}-a_{1}-1\right) / 2, \\
& a_{5}-a_{3}+1-\sum_{i=1,2,4}\left[\lambda+2-a_{i}-a_{3}\right]_{+}=a_{5}+1-\left(a_{2}+a_{3}+a_{4}-a_{1}-1\right) / 2, \\
& a_{5}-a_{2}+1-\sum_{i=1,3,4}\left[\lambda+2-a_{i}-a_{2}\right]_{+}=a_{5}+1-\left(a_{2}+a_{3}+a_{4}-a_{1}-1\right) / 2, \\
& a_{5}-a_{1}+1-\sum_{i=2,3,4}\left[\lambda+2-a_{i}-a_{1}\right]_{+}=a_{5}+2-\left(a_{2}+a_{3}+a_{4}-a_{1}-1\right) / 2, \\
& 2 a_{5}-\lambda-\sum_{1 \leq i<j \leq 4} A_{i j}=2 a_{5}+4-a_{4}-a_{3}-a_{2}+a_{1}=: 2 b .
\end{aligned}
$$

The additional hypothesis $2 a_{5}+3-a_{4}-a_{3}-a_{2}+a_{1} \geq 0$ guarantees that the orders of vanishing are positive. Therefore, applying Lemma 4.1, we obtain

$$
\begin{aligned}
\operatorname{dim}_{k}[R /(I, \ell)]_{\lambda} & =\operatorname{dim}_{k} \mathscr{L}_{2}\left(2 b ; b,\left(a_{5}+1-\left(a_{2}+a_{3}+a_{4}-a_{1}-1\right) / 2\right)^{3}, 1\right) \\
& \geq 2 a_{5}+5-\left(a_{2}+a_{3}+a_{4}-a_{1}\right)-1 .
\end{aligned}
$$

The hypothesis also guarantees that this value is positive. The computation of the "expected" dimension is very similar to what we did above. The extra hypothesis $a_{1}+a_{4}+3 \leq a_{2}+a_{3}$ implies that only $\lambda-a_{1}-a_{2}+2, \lambda-a_{1}-a_{3}+2$ and $\lambda-a_{1}-a_{4}+2$ are $>0$. We again have $a_{4}<a_{1}+a_{2}+a_{3}$. Thus, Lemma 3.7 provides

$$
\begin{aligned}
{\left[\operatorname{dim}_{k}[R / I]_{\lambda}-\operatorname{dim}_{k}[R / I]_{\lambda-1]}\right]_{+} } \\
=\left[h_{A}(\lambda)-h_{A}\left(\lambda-a_{5}\right)-h_{A}(\lambda-1)+h_{A}\left(\lambda-a_{5}-1\right)\right]_{+} \\
\quad=\left[\left(\begin{array}{c}
\lambda+2 \\
2
\end{array}\right)-\sum_{i=1}^{4}\left(\begin{array}{c}
\lambda-a_{i}+2 \\
2
\end{array}\right)+\sum_{i=2}^{4}\left(\begin{array}{c}
\lambda-a_{1}-a_{i}+2 \\
2
\end{array}\right)-\left(\begin{array}{c}
\lambda-a_{5}+2 \\
2
\end{array}\right)\right]_{+} .
\end{aligned}
$$

If this is zero, we are done. Otherwise, a straightforward computation shows that $\operatorname{dim}_{k}[R /(I, \ell)]_{\lambda}-\left[\operatorname{dim}_{k}[R / I]_{\lambda}-\operatorname{dim}_{k}[R / I]_{\lambda-1}\right]$ is given by

$$
\begin{array}{r}
2 a_{5}+4-\left(a_{2}+a_{3}+a_{4}-a_{1}\right) \\
-\left(\left(\begin{array}{c}
\lambda+2 \\
2
\end{array}\right)-\sum_{i=1}^{4}\left(\begin{array}{c}
\lambda-a_{i}+2 \\
2
\end{array}\right)+\sum_{i=2}^{4}\left(\begin{array}{c}
\lambda-a_{1}-a_{i}+2 \\
2
\end{array}\right)-\left(\begin{array}{c}
\lambda-a_{5}+2 \\
2
\end{array}\right)\right) \\
\quad=\left(\begin{array}{c}
\lambda-a_{5} \\
2
\end{array}\right)>0 .
\end{array}
$$


Thus in either case the actual value of $\operatorname{dim}[R /(I, \ell)]_{\lambda}$ exceeds the expected dimension. This completes the proof of the case $a_{1}+a_{4}+3 \leq a_{2}+a_{3}$.

Finally, we assume that $a_{1}+a_{4}+1=a_{2}+a_{3}$. We note first that this assumption actually forces the stronger condition $a_{1} \geq 4$ since $a_{4} \leq a_{5}<\lambda-1$ implies

$$
7<a_{1}+a_{2}+a_{3}-a_{4}=2 a_{1}+1,
$$

and hence $a_{1}>3$. The condition $a_{1}+a_{4}+1=a_{2}+a_{3}$ implies that $\lambda-a_{1}-a_{4}+2=0$, so the computation of the expected dimension above can only get smaller, while the computation of $\operatorname{dim}[R /(I, \ell)]_{\lambda}$ remains unchanged. Thus the difference can only grow, and we again have shown the failure of the WLP. This completes the proof of (iii).

In the previous results we excluded the case $a_{1}=2$ for the most part. The reason is that then the algebra does have the WLP.

Theorem 4.6. Let $L$ be a general linear form in $R:=k\left[x_{1}, \ldots, x_{4}\right]$, and let $I$ be the ideal $\left\langle x_{1}^{2}, x_{2}^{a_{2}}, x_{3}^{a_{3}}, x_{4}^{a_{4}}, L^{a_{5}}\right\rangle$ of $R$. Then the algebra $R / I$ has the WLP.

The proof will be based on a result about almost complete intersections in three variables generated by powers of four general linear forms. According to [Schenck and Seceleanu 2010] such an algebra has the WLP, that is, multiplication by a general linear form has maximal rank. We show that this is also true when one multiplies by the square of such a form.

Proposition 4.7. Let $\ell_{2}, \ldots, \ell_{5}, \ell$ be five general linear forms of $S:=k[x, y, z]$, and let $\mathfrak{a} \subset S$ be the ideal $\left\langle\ell_{2}^{a_{2}}, \ldots, \ell_{5}^{a_{5}}\right\rangle$. Set $A:=S / \mathfrak{a}$. Then, for each integer $j$, the multiplication map $\times \ell^{2}:[A]_{j-2} \rightarrow[A]_{j}$ has maximal rank.

Proof. If any of the numbers $a_{2}, \ldots, a_{5}$ equals one, then $A$ has the SLP by [Harima et al. 2003], so the claim is true. Thus, we may assume $2 \leq a_{2} \leq \cdots \leq a_{5}$.

First, assume also that

$$
a_{5} \leq \frac{a_{2}+a_{3}+a_{4}-3}{2} .
$$

Define integers $p$ and $b$ by

$$
p:=\left\lfloor\frac{a_{2}+a_{3}+a_{4}+a_{5}-4}{3}\right\rfloor \text { and } a_{2}+a_{3}+a_{4}+a_{5}=3(p+1)+b,
$$

thus $1 \leq b \leq 3$.

According to [Schenck and Seceleanu 2010], $A$ has the WLP and $A / \ell A$ has socle degree $p$. This implies that multiplication by $\ell$ on $A$ is injective until degree $p$ and surjective in larger degrees. Symbolically, this reads as

$$
[A]_{0} \hookrightarrow \cdots \hookrightarrow[A]_{p-1} \hookrightarrow[A]_{p} \rightarrow[A]_{p+1} \rightarrow \cdots
$$


Hence, to show our claim it suffices to prove that the multiplication

$$
\times \ell^{2}:[A]_{p-1} \rightarrow[A]_{p+1}
$$

has maximal rank, which is equivalent to

$$
\operatorname{dim}_{k}\left[A / \ell^{2} A\right]_{p+1}=\max \left\{0, h_{A}(p+1)-h_{A}(p-1)\right\} .
$$

In order to see this, we first compute the left-hand side and then the right-hand side. Using Theorem 3.3, we get

$$
\begin{aligned}
\operatorname{dim}_{k}\left[A / \ell^{2} A\right]_{p+1} & =\operatorname{dim}_{k}\left[S /\left\langle\ell^{2}, \ell_{2}^{a_{2}}, \ldots, \ell_{5}^{a_{5}}\right\rangle\right]_{p+1} \\
& =\operatorname{dim}_{k}\left[\mathfrak{p}^{p} \cap \mathfrak{p}_{2}^{p-a_{2}+2} \cap \ldots \cap \mathfrak{p}_{5}^{p-a_{5}+2}\right]_{p+1} \\
& =\operatorname{dim}_{k} \mathscr{L}_{2}\left(p+1 ; p, p-a_{2}+2, \ldots, p-a_{5}+2\right),
\end{aligned}
$$

where $\mathfrak{p}, \mathfrak{p}_{2}, \ldots, \mathfrak{p}_{5}$ are the homogenous ideals of five general points in $\mathbb{P}^{2}$. Let $Q \in S$ be the unique quadric that vanishes at these five points. Again, we use Bézout's theorem to estimate the multiplicity of $Q$ in the base locus of the linear system

$$
\mathscr{L}_{2}\left(p+1 ; p, p-a_{2}+2, \ldots, p-a_{5}+2\right) .
$$

For an integer $j$, the condition

$$
\begin{aligned}
2(p+1-2 j) & \geq p-j+\sum_{i=2}^{5}\left[p-a_{i}+2-j\right] \\
& =5 p+8-5 j-\sum_{i=2}^{5} a_{i} \\
& =5 p+8-5 j-[3(p+1)+b] \\
& =2 p+5-5 j-b
\end{aligned}
$$

is equivalent to $j \geq 3-b$. It follows that $Q$ appears with multiplicity at least $3-b$ in the base locus. Thus, we get

$$
\begin{array}{r}
\operatorname{dim}_{k}\left[A / \ell^{2} A\right]_{p+1}=\operatorname{dim}_{k} \mathscr{L}_{2}\left(p+1 ; p, p-a_{2}+2, \ldots, p-a_{5}+2\right) \\
=\operatorname{dim}_{k} \mathscr{L}_{2}\left(p+2 b-5 ; p+b-3, p+b-a_{2}-1,\right. \\
\left.\ldots, p+b-a_{5}-1\right) .
\end{array}
$$

The latter linear system is clearly empty if $b=1$. To compute its dimension if $2 \leq b \leq 3$, we consider the lines $L_{i}, 2 \leq i \leq 5$, passing through the points $\mathfrak{p}$ and $\mathfrak{p}_{i}$. By Bézout's theorem, the line $L_{i}$ appears with multiplicity at least $B_{i}$ in the base locus, where

$$
B_{i}:=\left[p+b-3+p+b-a_{i}-1-\{p+2 b-5\}\right]_{+}=\left[p+1-a_{i}\right]_{+} .
$$


Our assumption $a_{5} \leq\left(a_{2}+a_{3}+a_{4}-3\right) / 2$ implies $a_{5} \leq p+1$. Thus, we get $B_{i}=p+1-a_{i}$, so

$B_{2}+\cdots+B_{5}=4 p+4-\left[a_{2}+a_{3}+a_{4}+a_{5}\right]=4 p+4-[3(p+1)+b]=p+1-b$.

Removing the lines from the base locus we obtain

$$
\begin{array}{r}
\operatorname{dim}_{k}\left[A / \ell^{2} A\right]_{p+1}=\operatorname{dim}_{k} \mathscr{L}_{2}\left(p+2 b-5 ; p+b-3, p+b-a_{2}-1,\right. \\
\left.\ldots, p+b-a_{5}-1\right) \\
=\operatorname{dim}_{k} \mathscr{L}_{2}(3 b-6 ; 2 b-4, b-2, \ldots, b-2) .
\end{array}
$$

It follows that $\operatorname{dim}_{k}\left[A / \ell^{2} A\right]_{p+1}=1$ if $b=2$.

If $b=3$, then we get, using Theorem 3.3 again,

$$
\begin{aligned}
\operatorname{dim}_{k}\left[A / \ell^{2} A\right]_{p+1} & =\operatorname{dim}_{k} \mathscr{L}_{2}(3 ; 2,1, \ldots, 1) \\
& =\operatorname{dim}_{k}\left[S /\left\langle\ell^{2}, \ell_{2}^{3}, \ldots, \ell_{5}^{3}\right\rangle\right]_{3} \\
& =\operatorname{dim}_{k}\left[S /\left\langle\ell^{2}\right\rangle\right]_{3}-4 \\
& =3,
\end{aligned}
$$

because the linear forms are general. Summarizing, we have shown so far that

$$
\operatorname{dim}_{k}\left[A / \ell^{2} A\right]_{p+1}= \begin{cases}0 & \text { if } b=1 \\ 1 & \text { if } b=2 \\ 3 & \text { if } b=3\end{cases}
$$

Now we compute the right-hand side of (4-17). To this end consider the ring $B:=S /\left\langle\ell_{3}^{a_{3}}, \ell_{4}^{a_{4}}, \ell_{5}^{a_{5}}\right\rangle$. Observe that

$$
\begin{aligned}
p+1-a_{3}-a_{4} & =\left\lfloor\frac{a_{2}+a_{3}+a_{4}+a_{5}-1}{3}\right\rfloor-a_{3}-a_{4} \\
& \leq \frac{a_{2}+a_{5}-1-2 a_{3}-2 a_{4}}{3} \\
& \leq \frac{3 a_{2}-5-3 a_{3}-3 a_{4}}{6} \\
& <0,
\end{aligned}
$$

where we used again $a_{5} \leq\left(a_{2}+a_{3}+a_{4}-3\right) / 2$. Hence, the Koszul resolution of the complete intersection $B$ provides for its Hilbert function if $j \leq p+1$ that

$$
h_{B}(j)=\left(\begin{array}{c}
j+2 \\
2
\end{array}\right)-\sum_{i=3}^{5}\left(\begin{array}{c}
j+2-a_{i} \\
2
\end{array}\right) \text {, }
$$


where, as above, we define a binomial coefficient $\left(\begin{array}{l}c \\ 2\end{array}\right)$ to be zero if $c<0$. Since the complete intersection $B$ has the SLP and since $A \cong B / \ell_{2}^{a_{2}} B$, we get

$$
\begin{aligned}
h_{A}(p+1)- & h_{A}(p-1) \\
& =\left[h_{B}(p+1)-h_{B}\left(p+1-a_{2}\right)\right]_{+}-\left[h_{B}(p-1)-h_{B}\left(p-1-a_{2}\right)\right]_{+} .
\end{aligned}
$$

One easily checks that our assumptions provide $p+1 \leq\left\lceil\left(a_{3}+a_{4}+a_{5}-3\right) / 2\right\rceil$. Since $a_{3}+a_{4}+a_{5}-3$ is the socle degree of $B$, this implies that $h_{B}(j-1) \leq h_{B}(j)$ if $j \leq p+1$. Hence the last formula simplifies to

$h_{A}(p+1)-h_{A}(p-1)=h_{B}(p+1)-h_{B}\left(p+1-a_{2}\right)-h_{B}(p-1)+h_{B}\left(p-1-a_{2}\right)$.

The socle degree of $A / \ell A$ is at most the socle degree of $S /\left\langle\ell, \ell_{2}^{a_{2}}, \ell_{3}^{a_{3}}\right\rangle$, thus $p \leq a_{2}+a_{3}-2$. Combining with (4-19) and using that

$$
\left(\begin{array}{c}
j+1 \\
2
\end{array}\right)-\left(\begin{array}{c}
j-1 \\
2
\end{array}\right)=2 j-1
$$

if $j \geq 1$, this provides

$$
\begin{aligned}
h_{A}(p+1)-h_{A}(p-1) & =\left(\begin{array}{c}
p+3 \\
2
\end{array}\right)-\left(\begin{array}{c}
p+1 \\
2
\end{array}\right)-\sum_{i=3}^{5}\left[\left(\begin{array}{c}
p+3-a_{a} \\
2
\end{array}\right)-\left(\begin{array}{c}
p+1-a_{i} \\
2
\end{array}\right)\right] \\
& -\left[\left(\begin{array}{c}
p+3-a_{2} \\
2
\end{array}\right)-\left(\begin{array}{c}
p+1-a_{2} \\
2
\end{array}\right)\right] \\
& =-6 p-9+2 \sum_{i=2}^{4} a_{i} \\
& =2 b-3 .
\end{aligned}
$$

Hence, we get

$$
\left[h_{A}(p+1)-h_{A}(p-1)\right]_{+}=[2 b-3]_{+}= \begin{cases}0 & \text { if } b=1, \\ 1 & \text { if } b=2, \\ 3 & \text { if } b=3 .\end{cases}
$$

Comparing with (4-18), this establishes the desired equality (4-17).

It remains to consider the case $a_{5}>\left(a_{2}+a_{3}+a_{4}-3\right) / 2$. Let us call $\mathfrak{b}:=$ $\left\langle\ell_{2}^{a_{2}}, \ell_{3}^{a_{3}}, \ell_{4}^{a_{4}}\right\rangle$ and set $B:=S / \mathfrak{b}$. Note that the socle degree of $B$ is $a_{2}+a_{3}+a_{4}-3$. Stanley [1980], Watanabe [1987] and Reid, Roberts and Roitman [1991] showed that, in characteristic 0 , SLP holds for an artinian complete intersection generated by powers of linear forms. In particular, for each integer $j$, the multiplication map $\times \ell^{2}:[B]_{j-2} \rightarrow[B]_{j}$ has maximal rank. We want to prove that, for each integer $j$, the multiplication map $\times \ell^{2}:[A]_{j-2} \rightarrow[A]_{j}$ has maximal rank. To this end, we will examine several cases:

(a) Assume $a_{2}+a_{3}+a_{4}$ odd. 
(a1) For any $j<a_{5}$, we have $[A]_{j} \cong[B]_{j}$ and hence $\times \ell^{2}:[A]_{j-2} \rightarrow[A]_{j}$ has maximal rank.

(a2) For any $j \geq a_{5}+1$ or $j=a_{5}$ and $a_{5}>\left(a_{2}+a_{3}+a_{4}-3\right) / 2+1$, we have $j-2 \geq\left(a_{2}+a_{3}+a_{4}-3\right) / 2$. Hence, the surjectivity $\times \ell^{2}:[B]_{j-2} \rightarrow[B]_{j}$ together with the commutative diagram

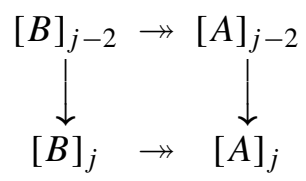

allows us to conclude that $\times \ell^{2}:[A]_{j-2} \rightarrow[A]_{j}$ has maximal rank.

(a3) For $j=a_{5}=\left(a_{2}+a_{3}+a_{4}-3\right) / 2+1$ we have $[A]_{j-2} \cong[B]_{j-2} \cong[B]_{j} \rightarrow[A]_{j}$. Therefore, we also conclude that $\times \ell^{2}:[A]_{j-2} \rightarrow[A]_{j}$ has maximal rank.

(b) Assume $a_{2}+a_{3}+a_{4}$ even.

(b1) For any $j<a_{5}$, we have $[A]_{j} \cong[B]_{j}$ and hence $\times \ell^{2}:[A]_{j-2} \rightarrow[A]_{j}$ has maximal rank.

(b2) It is identical to (a2).

(b3) For $j=a_{5}=\left(a_{2}+a_{3}+a_{4}-2\right) / 2$ we have to prove that $\times \ell^{2}:[A]_{j-2} \rightarrow[A]_{j}$ is injective. Since $\operatorname{dim}[B]_{j-2}<\operatorname{dim}[B]_{j}$, the image of $[B]_{j-2}$ in $[B]_{j}$ under multiplication by $\ell^{2}$ is not surjective. So the vector space $\left[\left\langle\ell_{2}^{a_{2}}, \ell_{3}^{a_{3}}, \ell_{4}^{a_{4}}, \ell^{2}\right\rangle\right]_{j}$ is not all of $S_{j}$. But in characteristic zero the $j$-th powers of linear forms span $[S]_{j}$. Thus for a general $L$, the image of $L^{j}=L^{a_{5}}$ in $[B]_{j}$ is outside the image of $[B]_{j-2}$ in $[B]_{j}$. Thus $\left(\times \ell^{2}\right):[A]_{j-2} \rightarrow[A]_{j}$ is injective, since it is for $B$ in that degree. This completes the proof of the proposition.

Remark 4.8. Observe that extensions of Proposition 4.7 to multiplication by higher powers of $\ell$ fail in general. There are many such examples. The smallest is when $a_{2}=\cdots=a_{5}=3$, for which multiplication by $\ell^{3}$ fails to have maximal rank from degree 1 to degree 4 . It is easy to see that this extends to the case $a_{2}=\cdots=a_{5}=d$, for which multiplication by $\ell^{d}$ fails to have maximal rank from degree $d-2$ to degree $2 d-2$. Many more complicated examples (produced by CoCoA) exist as well.

Proof of Theorem 4.6. If one of the numbers $a_{2}, \ldots, a_{5}$ is one, then the result follows by [Schenck and Seceleanu 2010]. Thus, we may assume $2 \leq a_{2} \leq \cdots \leq a_{5}$.

If $a_{5}>\left(a_{2}+a_{3}+a_{4}-3\right) / 2$, then $R / I$ has the WLP by Theorems $4.2(\mathrm{i})$ and 4.5(i). Thus, we may assume $a_{5} \leq\left(a_{2}+a_{3}+a_{4}-3\right) / 2$ for the remainder of the proof. Let $\ell \in R$ be another general linear form. We have to show that for all integers $j$,

$$
\operatorname{dim}_{k}[R /(I, \ell)]_{j}=\max \left\{0, h_{R / I}(j)-h_{R / I}(j-1)\right\} .
$$


To this end, consider the ideal $J:=\left\langle x_{2}^{a_{2}}, x_{3}^{a_{3}}, x_{4}^{a_{4}}, L^{a_{5}}\right\rangle \subset R$. The complete intersection $R / J$ has the SLP, which implies

$$
\begin{aligned}
& {\left[h_{R / I}(j)-h_{R / I}(j-1)\right]_{+}} \\
& \quad=\left[\left[h_{R / J}(j)-h_{R / J}(j-2)\right]_{+}-\left[h_{R / J}(j-1)-h_{R / J}(j-3)\right]_{+}\right]_{+} .
\end{aligned}
$$

The complete intersection $R / J$ has socle degree $a_{2}+\cdots+a_{5}-4 \geq 4$, thus the multiplication $\times \ell:[R / J]_{j-1} \rightarrow[R / J]_{j}$ is injective if $j \leq\left\lceil\left(a_{2}+a_{3}+a_{4}+a_{5}-4\right) / 2\right\rceil$. One checks that

$$
\left\lceil\frac{a_{2}+a_{3}+a_{4}+a_{5}-4}{2}\right\rceil \geq\left\lfloor\frac{a_{2}+a_{3}+a_{4}+a_{5}-1}{3}\right\rfloor=: p+1 .
$$

It follows that $h_{R / J}(j-1) \leq h_{R / J}(j)$ if $j \leq p+1$. Thus, if $j \leq p+1$, then we get that

$$
\begin{aligned}
h_{R / I}(j)-h_{R / I}(j- & 1) \\
& =h_{R / J}(j)-h_{R / J}(j-2)-h_{R / J}(j-1)+h_{R / J}(j-3) \\
& =\left[h_{R / J}(j)-h_{R / J}(j-1)\right]_{+}-\left[h_{R / J}(j-2)-h_{R / J}(j-3)\right]_{+} \\
& =h_{R /(J, \ell)}(j)-h_{R /(J, \ell)}(j-2),
\end{aligned}
$$

where we used again that $R / J$ has the SLP. Invoking Proposition 4.7, we obtain

$$
\left[h_{R / I}(j)-h_{R / I}(j-1)\right]_{+}=h_{R /(I, \ell)}(j)
$$

if $j \leq p+1$. This equality is also true (using the same computations) if $h_{R / J}(p+1) \leq$ $h_{R / J}(p+2)$. Otherwise, we get $\left[h_{R / I}(p+2)-h_{R / I}(p+1)\right]_{+}=0$. However, using (4-16), we get $[R /(I, \ell)]_{p+2}=0$. Hence, we have in any case

$$
\left[h_{R / I}(p+2)-h_{R / I}(p+1)\right]_{+}=h_{R /(I, \ell)}(p+2)=0,
$$

which completes the argument.

\section{Almost uniform powers of linear forms in 5 variables}

In this section, we let $R=k\left[x_{1}, x_{2}, x_{3}, x_{4}, x_{5}\right]$, where $k$ is a field of characteristic zero, and we will apply the approach described in Section 3 and results on fat points in $\mathbb{P}^{3}$ to determine exactly when an ideal generated by uniform powers of six general linear forms in $R$ fails the WLP. Some nonuniform cases are also discussed. To this end, without loss of generality we set $I=\left\langle x_{1}^{a_{1}}, x_{2}^{a_{2}}, x_{3}^{a_{3}}, x_{4}^{a_{4}}, x_{5}^{a_{5}}, L^{a_{6}}\right\rangle$, where $L$ is a general linear form and $a_{1} \leq a_{2} \leq a_{3} \leq a_{4} \leq a_{5} \leq a_{6}$.

Theorem 5.1. Let $L$ be a general linear form and let $I=\left\langle x_{1}^{d}, x_{2}^{d}, x_{3}^{d}, x_{4}^{d}, x_{5}^{d}, L^{d}\right\rangle$. Then the ring $R / I$ fails the WLP if and only if $d>3$. 
Proof. Using CoCoA we check that if $d<4$ then $R / I$ has the WLP. Assume $d \geq 4$ and we will show that $R / I$ fails the WLP in degree $2 d-1$. To this end, we take $\ell \in R$ a general linear form. According to (3-1), it is enough to show that

$$
\operatorname{dim}[R /(I, \ell)]_{2 d-1}>\left[\operatorname{dim}[R / I]_{2 d-1}-\operatorname{dim}[R / I]_{2 d-2}\right]_{+} .
$$

We will compute the left-hand side using Proposition 3.4 and the right-hand side using the fact that $R / J$ has the SLP.

Now let $S=k\left[x_{1}, x_{2}, x_{3}, x_{4}\right]$. We want to compute the vector space dimension

$$
\operatorname{dim}_{k}\left[\mathfrak{q}_{1}^{d} \cap \mathfrak{q}_{2}^{d} \cap \mathfrak{q}_{3}^{d} \cap \mathfrak{q}_{4}^{d} \cap \mathfrak{q}_{5}^{d} \cap \mathfrak{q}_{6}^{d}\right]_{2 d-1}=\operatorname{dim}_{k} \mathscr{L}_{3}\left(2 d-1 ; d^{6}\right) .
$$

Applying a sequence of cubo-cubic Cremona transformations

$$
\left(x_{1}: x_{2}: x_{3}: x_{4}\right) \stackrel{\rightarrow-\rightarrow}{\rightarrow}\left(x_{1}^{-1}, x_{2}^{-1}, x_{3}^{-1}, x_{4}^{-1}\right)=\left(x_{2} x_{3} x_{4}: x_{1} x_{3} x_{4}: x_{1} x_{2} x_{4}: x_{1} x_{2} x_{3}\right)
$$

we will transform the last linear system to another one which has the same dimension, but it will be nonspecial and hence we will be able to compute its dimension. In fact, we apply Lemma 3.5 and we get

$$
\begin{aligned}
\operatorname{dim} \mathscr{L}_{3}\left(2 d-1 ; d^{6}\right) & =\operatorname{dim} \mathscr{L}_{3}\left(2 d-3 ; d^{2},(d-2)^{4}\right) \\
& =\operatorname{dim} \mathscr{L}_{3}\left(2 d-5 ;(d-2)^{4},(d-4)^{2}\right) \\
& =\operatorname{dim} \mathscr{L}_{3}\left(2 d-7 ;(d-4)^{6}\right) .
\end{aligned}
$$

Since $2(2 d-7) \geq 4(d-4)$, the linear system $\mathscr{L}_{3}\left(2 d-7 ;(d-4)^{6}\right)$ is in standard form. Therefore, Theorem 3.6 provides that it is nonspecial and its dimension is given by

$$
\operatorname{dim} \mathscr{L}_{3}\left(2 d-7 ;(d-4)^{6}\right)=\left(\begin{array}{c}
2 d-4 \\
3
\end{array}\right)-6\left(\begin{array}{c}
d-2 \\
3
\end{array}\right) .
$$

Now we have to compute the right-hand side of (5-1). Let $A=R / J$, where $J=\left\langle x_{1}^{d}, x_{2}^{d}, x_{3}^{d}, x_{4}^{d}, x_{5}^{d}\right\rangle$. Since $R / J$ has the SLP, we have

$$
0 \leq h_{R / I}(2 d-1)=h_{A}(2 d-1)-h_{A}(d-1) .
$$

Therefore,

$\left[\operatorname{dim}[R / I]_{2 d-1}-\operatorname{dim}[R / I]_{2 d-2}\right]$

$$
\begin{aligned}
& =h_{A}(2 d-1)-h_{A}(d-1)-h_{A}(2 d-2)+h_{A}(d-2) \\
& =\left(\begin{array}{c}
2 d+2 \\
3
\end{array}\right)-6\left(\begin{array}{c}
d+2 \\
3
\end{array}\right) .
\end{aligned}
$$

We easily verify that

$$
\operatorname{dim}[R /(I, \ell)]_{2 d-1}-\left[\operatorname{dim}[R / I]_{2 d-1}-\operatorname{dim}[R / I]_{2 d-2}\right]=4
$$


and this shows that $R / I$ fails the WLP in degree $2 d-1$, which is what we wanted to prove.

Remark 5.2. Note that (5-4) could also have been proven using Proposition 3.5 of [Catalisano et al. 1999]. However, we use our approach because it also applies to setups below where the hypotheses of the latter proposition are not satisfied.

There are several possible extensions of the above theorem. First, we can ask whether the WLP property holds for the case of nonuniform powers and, in particular, we can ask what happens in the almost uniform case. We have

Theorem 5.3. Let $L$ be a general linear form and let $I=\left\langle x_{1}^{d}, x_{2}^{d}, x_{3}^{d}, x_{4}^{d}, x_{5}^{d}, L^{d+e}\right\rangle$ with $e \geq 1$. Then:

(i) If $d$ is odd, then $R / I$ has the WLP if and only if $e \geq(3 d-5) / 2$.

(ii) If $d$ is even, $R / I$ has the WLP if and only if $e \geq(3 d-8) / 2$.

Proof. Set $\lambda:=\lfloor(5 d-5) / 2\rfloor$. We will actually prove the following sequence of statements:

(i') If $d+e \geq \lambda$, then $R / I$ has the WLP.

(ii') If $d$ is odd and $e \leq d-2$, then $R / I$ fails the WLP.

(iii') If $d$ is even and $e \leq d-3$, then $R / I$ fails the WLP.

(iv') If $d$ is odd and $d-1 \leq e \leq(3 d-7) / 2$ then $R / I$ fails the WLP.

$\left(\mathrm{v}^{\prime}\right)$ If $d$ is even and $d-2 \leq e \leq(3 d-10) / 2$ then $R / I$ fails the WLP.

(vi') If $d$ is even and $e=(3 d-8) / 2$ (that is $d+e=\lambda-1$ ) then $R / I$ has the WLP.

Throughout this proof we will denote $A=R / J$, where $J=\left\langle x_{1}^{d}, x_{2}^{d}, x_{3}^{d}, x_{4}^{d}, x_{5}^{d}\right\rangle$.

(i') The proof is the same as in Theorems 4.2(i) and 4.5(i).

(ii') Since $d+e \leq 2 d-2$, we can write $e=2 j+\epsilon$ with $0 \leq \epsilon \leq 1$ and $j \leq(d-3) / 2$. We will show that $R / I$ fails the WLP in degree $2 d-1+j$. To this end, we take $\ell \in R$, a general linear form. According to (3-1), it is enough to show that

$$
\operatorname{dim}[R /(I, \ell)]_{2 d-1+j}>\left[\operatorname{dim}[R / I]_{2 d-1+j}-\operatorname{dim}[R / I]_{2 d-2+j}\right]_{+} .
$$

We will compute the left-hand side using Proposition 3.4 and the right-hand side using Lemma 3.7.

We begin by computing the vector space dimension $\operatorname{dim}_{k}\left[\mathfrak{q}_{1}^{d+j} \cap \mathfrak{q}_{2}^{d+j} \cap \mathfrak{q}_{3}^{d+j} \cap \mathfrak{q}_{4}^{d+j} \cap \mathfrak{q}_{5}^{d+j} \cap \mathfrak{q}_{6}^{d+j-e}\right]_{2 d-1+j}$

$$
=\operatorname{dim}_{k} \mathscr{L}_{3}\left(2 d-1+j ;(d+j)^{5}, d+j-e\right) .
$$


Applying a sequence of cubo-cubic Cremona transformations (see Lemma 3.5), we get

$$
\begin{aligned}
\operatorname{dim}_{k} & \mathscr{L}_{3}\left(2 d-1+j ;(d+j)^{5}, d+j-e\right) \\
& =\operatorname{dim}_{k} \mathscr{L}_{3}(2 d-3-j ; d+j, d+j-e),(d-j-2)^{4} \\
& =\operatorname{dim}_{k} \mathscr{L}_{3}\left(2 d-5-3 j+e ; d-j-2+e,(d-j-2)^{3},(d-3 j-4+e)^{2}\right) \\
& =\operatorname{dim}_{k} \mathscr{L}_{3}\left(2 d-7-5 j+2 e ; d-3 j-4+2 e,(d-3 j-4+e)^{5}\right) .
\end{aligned}
$$

(Here we use the hypothesis $d+e \leq 2 d-2$ to guarantee that $d-3 j-4+2 e \geq 0$.)

Since $2(2 d-7-5 j+2 e) \geq 3(d-3 j-4+e)+d-3 j-4+2 e$, the linear system

$$
\mathscr{L}_{3}\left(2 d-7-5 j+2 e ; d-3 j-4+2 e,(d-3 j-4+e)^{5}\right)
$$

is in standard form. Therefore, Theorem 3.6 provides

$$
\begin{aligned}
& \operatorname{dim}_{k} \mathscr{L}_{3}\left(2 d-7-5 j+2 e ;(d-3 j-4+e)^{5}, d-3 j-4+2 e\right) \\
& \quad=\left[\left(\begin{array}{c}
2 d-4-5 j+2 e \\
3
\end{array}\right)-5\left(\begin{array}{c}
d-2-3 j+e \\
3
\end{array}\right)-\left(\begin{array}{c}
d-2-3 j+2 e \\
3
\end{array}\right)\right]_{+}+5 \cdot\left(\begin{array}{c}
e-j \\
3
\end{array}\right) .
\end{aligned}
$$

We claim that

$$
\begin{aligned}
& \left(\begin{array}{c}
2 d-4-5 j+2 e \\
3
\end{array}\right)-5\left(\begin{array}{c}
d-2-3 j+e \\
3
\end{array}\right)-\left(\begin{array}{c}
d-2-3 j+2 e \\
3
\end{array}\right)+5 \cdot\left(\begin{array}{c}
e-j \\
3
\end{array}\right) \\
& =\frac{1}{6}(d-2-2 j)\left[2 d^{2}-3 e^{2}+12 e(1+j)-4\left(3+8 j+4 j^{2}\right)+d(3 e-2-2 j)\right]
\end{aligned}
$$

is positive. In fact, using $d \geq 2 j+3$ and $e \geq 2 e$, one gets

$$
\begin{aligned}
& 2 d^{2}-3 e^{2}+12 e(1+j)-4\left(3+8 j+4 j^{2}\right)+d(3 e-2-2 j) \\
& \geq 2(2 j+3)^{2}-3 e^{2}+12 e(1+j)-4\left(3+8 j+4 j^{2}\right)+(2 j+3)(3 e-2-2 j)>0 .
\end{aligned}
$$

We conclude that $\operatorname{dim}[R /(I, \ell)]_{2 d-1+j}>0$.

Now we compute the right-hand side of (5-5). Lemma 3.7 provides

$$
\begin{aligned}
\operatorname{dim} & {[R / I]_{2 d-1+j}-\operatorname{dim}[R / I]_{2 d-2+j} } \\
& =h_{A}(2 d-1+j)-h_{A}(d-1+j-e)-h_{A}(2 d-2+j)+h_{A}(d-2+j-e) \\
& =\left(\begin{array}{c}
2 d+j+2 \\
3
\end{array}\right)-5\left(\begin{array}{c}
d+j+2 \\
3
\end{array}\right)-\left(\begin{array}{c}
d+j+2-e \\
3
\end{array}\right)+10\left(\begin{array}{c}
j+2 \\
3
\end{array}\right) .
\end{aligned}
$$

If $\operatorname{dim}[R / I]_{2 d-1+j}-\operatorname{dim}[R / I]_{2 d-2+j} \leq 0$, then the WLP fails because we have seen that $\operatorname{dim}[R /(I, \ell)]_{2 d-1+j}>0$. 
If $\operatorname{dim}[R / I]_{2 d-1+j}-\operatorname{dim}[R / I]_{2 d-2+j}>0$, then

$$
\begin{aligned}
& \operatorname{dim}[R /(I, \ell)]_{2 d-1+j}-\left[\operatorname{dim}[R / I]_{2 d-1+j}-\operatorname{dim}[R / I]_{2 d-2+j}\right] \\
& \geq\left(\begin{array}{c}
2 d-4-5 j+2 e \\
3
\end{array}\right)-5\left(\begin{array}{c}
d-2-3 j+e \\
3
\end{array}\right)-\left(\begin{array}{c}
d-2-3 j+2 e \\
3
\end{array}\right)+5 \cdot\left(\begin{array}{c}
e-j \\
3
\end{array}\right) \\
& \quad-\left[\left(\begin{array}{c}
2 d+j+2 \\
3
\end{array}\right)-5\left(\begin{array}{c}
d+j+2 \\
3
\end{array}\right)-\left(\begin{array}{c}
d+j+2-e \\
3
\end{array}\right)+10\left(\begin{array}{c}
j+2 \\
3
\end{array}\right)\right] \\
& =\left(\begin{array}{c}
3 j-e+4 \\
3
\end{array}\right)>0 .
\end{aligned}
$$

Hence, we conclude in every case that $R / I$ fails the WLP in degree $2 d-1+j$, which is what we wanted to prove.

(iii') The proof is completely analogous.

(iv') Suppose that $d$ is odd and $d-1 \leq e \leq(3 d-7) / 2$. We claim that $R / I$ fails the WLP (usually by failing injectivity) from degree $(5 d-7) / 2$ to degree $(5 d-5) / 2$. We first consider (by applying a sequence of cubo-cubic Cremona transformations; see Lemma 3.5)

$$
\begin{aligned}
\operatorname{dim}[R /(I, \ell)]_{(5 d-5) / 2} & =\operatorname{dim}_{k} \mathscr{L}\left(\frac{5 d-5}{2} ;\left(\frac{3 d-3}{2}\right)^{5}, \frac{3 d-3}{2}-e\right) \\
& =\operatorname{dim}_{k} \mathscr{L}_{3}\left(\frac{3 d-3}{2} ;\left(\frac{d-1}{2}\right)^{4}, \frac{3 d-3}{2}, \frac{3 d-3}{2}-e\right) \\
& =\operatorname{dim}_{k} \mathscr{L}_{3}\left(\frac{3 d-3}{2} ; \frac{3 d-3}{2},\left(\frac{d-1}{2}\right)^{4}, \frac{3 d-3}{2}-e\right) \\
& =\operatorname{dim}_{k} \mathscr{L}_{2}\left(\frac{3 d-3}{2} ;\left(\frac{d-1}{2}\right)^{4}, \frac{3 d-3}{2}-e\right),
\end{aligned}
$$

the last step being a consequence of Proposition 3.4. Notice that since $d-1 \leq e$, we have $(3 d-3) / 2-e \leq(d-1) / 2$. Thanks to Lemma 4.1, these fat points impose independent conditions, so we have

$$
\begin{aligned}
\operatorname{dim}[R /(I, \ell)]_{(5 d-5) / 2} & =\left(\begin{array}{c}
\frac{3 d-3}{2}+2 \\
2
\end{array}\right)-4 \cdot\left(\begin{array}{c}
\frac{d-1}{2}+1 \\
2
\end{array}\right)-\left(\begin{array}{c}
\frac{3 d-3}{2}-e+1 \\
2
\end{array}\right) \\
& =\frac{9 d^{2}-1}{8}-4 \cdot \frac{d^{2}-1}{8}-\frac{1}{2}\left[\frac{9 d^{2}-12 d+3}{4}-e(3 d-2)+e^{2}\right] \\
& =\frac{e}{2}(3 d-2-e)-\left(\begin{array}{c}
d-1 \\
2
\end{array}\right)+1
\end{aligned}
$$

(The fact that

$$
\begin{aligned}
\operatorname{dim}_{k} \mathscr{L}_{3}\left(\frac{3 d-3}{2} ; \frac{3 d-3}{2}, \frac{d-1}{2}, \frac{d-1}{2}, \frac{d-1}{2}, \frac{d-1}{2},\right. & \left.\frac{3 d-3}{2}-e\right) \\
& =\frac{e}{2}(3 d-2-e)-\left(\begin{array}{c}
d-1 \\
2
\end{array}\right)+1
\end{aligned}
$$

could also be obtained via Theorem 3.6.) 
Combined with Lemma 3.7, we obtain $\operatorname{dim}[R /(I, \ell)]_{(5 d-5) / 2}-\left(\operatorname{dim}[R / I]_{(5 d-5) / 2}-\operatorname{dim}[R / I]_{((5 d-5) / 2)-1}\right)$

$$
=\left(\begin{array}{c}
\frac{3 d-7}{2}-e+3 \\
3
\end{array}\right)
$$

If the part in parentheses is nonnegative then we expect injectivity, but the positivity of the part on the right (since we assumed $e \leq(3 d-7) / 2)$ implies that the WLP fails. Now suppose that the part in parentheses is negative, so that we expect surjectivity. Then one checks that

$$
\begin{aligned}
\operatorname{dim}[R /(I, \ell)]_{(5 d-5) / 2} & =\frac{e}{2}(3 d-2-e)-\left(\begin{array}{c}
d-1 \\
2
\end{array}\right)+1 \\
& =\frac{(e-d)(2 d-e-2)}{2}+\left(\begin{array}{c}
d+1 \\
2
\end{array}\right) \\
& \geq \frac{(e-d)(d+3)}{4}+\left(\begin{array}{c}
d+1 \\
2
\end{array}\right) \quad\left(\text { since } e \leq \frac{3 d-7}{2}\right) \\
& >0 .
\end{aligned}
$$

(the last inequality is for $d-1 \leq e \leq(3 d-7) / 2)$, and so surjectivity, and hence WLP, fails. This completes the proof of (iv').

$\left(\mathrm{v}^{\prime}\right)$ We show that $R / I$ fails the WLP from degree $(5 d-8) / 2$ to degree $(5 d-6) / 2$. Since we will use part of the computations also for (vi'), we consider all even $d$ such that $d-2 \leq e \leq(3 d-8) / 2$. In the same way as above we get

$$
\begin{aligned}
\operatorname{dim}[R /(I, \ell)]_{(5 d-6) / 2} & =\operatorname{dim}_{k} \mathscr{L}_{3}\left(\frac{5 d-6}{2} ;\left(\frac{3 d-4}{2}\right)^{5}, \frac{3 d-4}{2}-e\right) \\
& =\operatorname{dim}_{k} \mathscr{L}_{3}\left(\frac{3 d-2}{2} ;\left(\frac{d}{2}\right)^{4}, \frac{3 d-4}{2}, \frac{3 d-4}{2}-e\right) .
\end{aligned}
$$

Using Theorem 3.6, we obtain

$$
\operatorname{dim}[R /(I, \ell)]_{(5 d-6) / 2}=-d^{2}+3 d e-e^{2}+6 d-4 e-4=\frac{5 d^{2}}{4}-\left(\frac{3 d-4}{2}-e\right)^{2},
$$

which is at least $d^{2}$ in the given range for $e$. In particular, we are finished whenever $\operatorname{dim}[R / I]_{(5 d-6) / 2} \leq \operatorname{dim}[R / I]_{((5 d-6) / 2)-1}$.

If $\operatorname{dim}[R / I]_{(5 d-6) / 2} \geq \operatorname{dim}[R / I]_{((5 d-6) / 2)-1}$ (so we expect injectivity), then Lemma 3.7 provides, minding the bound on $e$,

$$
\begin{aligned}
\operatorname{dim}[R /(I, \ell)]_{(5 d-6) / 2}-\left(\operatorname{dim}[R / I]_{(5 d-6) / 2}-\operatorname{dim}[R / I]_{((5 d-6) / 2)-1}\right) & \\
& =\left(\begin{array}{c}
\frac{3 d-10}{2}-e+3 \\
3
\end{array}\right) .
\end{aligned}
$$

Since we are assuming that the part in parentheses is nonnegative, the positivity of the part on the right implies that WLP fails if $e \leq(3 d-10) / 2$, as claimed. 
(vi') Arguing as in Theorem 4.5(iii), we see that if $t \leq \lambda-2$ or $t \geq \lambda$ then $(\times \ell)$ : $[R / I]_{t} \rightarrow[R / I]_{t+1}$ has maximal rank. So, it only remains to study the case $t=\lambda-1$. We are going to prove that the multiplication $(\times \ell):[R / I]_{\lambda-1} \rightarrow[R / I]_{\lambda}$ is injective, that is $\operatorname{dim}[R /(I, \ell)]_{\lambda}=\operatorname{dim}[R / I]_{\lambda}-\operatorname{dim}[R / I]_{\lambda-1}$.

Notice that the assumptions force $d \geq 4$, so we may apply the computations of $\left(v^{\prime}\right)$. Thus, the desired follows by (5-6).

The same argument gives us the following result.

Proposition 5.4. Let $L$ be a general linear form and let

$$
I=\left\langle x_{1}^{a_{1}}, x_{2}^{a_{2}}, x_{3}^{a_{3}}, x_{4}^{a_{4}}, x_{5}^{a_{5}}, L^{a_{6}}\right\rangle .
$$

Assume that $5 \leq a_{1} \leq a_{2} \leq a_{3} \leq a_{4} \leq a_{5} \leq a_{6} \leq a_{1}+2$. Then the ring $R / I$ fails the $W L P$.

Our methods extend beyond the results mentioned above.

Example 5.5. Using the above notation, one shows:

(i) If $d \geq 4$ and $\left(a_{1}, a_{2}, a_{3}, a_{4}, a_{5}, a_{6}\right)=(d, d+1, d+2, d+3, d+4, d+5)$, then the ring $R / I$ fails the WLP in degree $2 d+4$.

(ii) If $d \geq 4$ and $\left(a_{1}, a_{2}, a_{3}, a_{4}, a_{5}, a_{6}\right)=(d, d+3, d+4, d+7, d+7, d+10)$, then the ring $R / I$ fails the WLP in degree $2 d+9$.

\section{Uniform powers of linear forms}

In this section, we consider the case of an almost complete intersection of general linear forms whose generators all have the same degree $d$. We give a complete answer, in the case of an even number of variables, to the question of when the WLP holds. Interestingly, the case of an odd number of variables is more delicate, and we are only able to give a partial result, concluding with a conjecture.

We first consider the case where there are an even number of variables. Set $R=k\left[x_{1}, \ldots, x_{2 n}\right]$, where $k$ is a field of characteristic zero. We determine when an ideal generated by uniform powers of $2 n+1$ general linear forms in $R$ fails the WLP. If $n=1$, then $R / I$ always has the WLP due to [Harima et al. 2003]. If $n=2$, then $R / I$ fails the WLP if and only if $d \geq 3$ by Theorem 4.2.

Theorem 6.1. Let $L \in R$ be a general linear form, and let $I=\left\langle x_{1}^{d}, \ldots, x_{2 n}^{d}, L^{d}\right\rangle$, where $n \geq 3$. Then the ring $R / I$ fails the WLP if and only if $d>1$.

Proof. It is clear that for $d=1, R / I$ has WLP.

Assume $d \geq 2$. We will show that $R / I$ fails WLP in degree $n d-n$. To this end, we take $\ell \in R$, a general linear form. According to (3-1), it is enough to show that

$$
\operatorname{dim}_{k}[R /(I, \ell)]_{n d-n}>\left[\operatorname{dim}_{k}[R / I]_{n d-n}-\operatorname{dim}_{k}[R / I]_{n d-n-1}\right]_{+} .
$$


First, we compute the left-hand side of (6-1).

Claim 1. $\operatorname{dim}[R /(I, \ell)]_{n d-n}=1$.

Proof. By Proposition 3.4 and Theorem 3.3, we have

$$
\begin{aligned}
& \operatorname{dim}_{k}[R /(I, \ell)]_{n d-n} \\
& \quad=\operatorname{dim}_{k}\left[\mathfrak{q}_{1}^{(n-1) d-(n-1)} \cap \mathfrak{q}_{2}^{(n-1) d-(n-1)} \cap \cdots \cap \mathfrak{q}_{2 n}^{(n-1) d-(n-1)} \cap \mathfrak{q}_{2 n+1}^{(n-1) d-(n-1)}\right]_{n d-n} \\
& \quad=\operatorname{dim}_{k} \mathscr{L}_{2 n-2}\left(n d-n ;((n-1) d-(n-1))^{2 n+1}\right) .
\end{aligned}
$$

Applying Lemma 3.5, we get

$\operatorname{dim}_{k} \mathscr{L}_{2 n-2}\left(n d-n ;((n-1) d-(n-1))^{2 n+1}\right)$

$=\operatorname{dim}_{k} \mathscr{L}_{2 n-2}\left((n-1) d-(n-1) ;((n-1) d-(n-1))^{2},((n-2) d-(n-2))^{2 n-1}\right)$.

Using Proposition 3.4 twice, it follows that

$$
\begin{aligned}
& \operatorname{dim}_{k} \mathscr{L}_{2 n-2}\left(n d-n ;((n-1) d-(n-1))^{2 n+1}\right) \\
& \quad=\operatorname{dim}_{k} \mathscr{L}_{2 n-3}\left((n-1) d-(n-1) ;(n-1) d-(n-1),((n-2) d-(n-2))^{2 n-1}\right) \\
& \quad=\operatorname{dim}_{k} \mathscr{L}_{2 n-4}\left((n-1) d-(n-1) ;((n-2) d-(n-2))^{2 n-1}\right) .
\end{aligned}
$$

If $n=3$, then we get by applying again Lemma 3.5

$$
\operatorname{dim}_{k} \mathscr{L}_{2}\left(2 d-2 ;(d-1)^{5}\right)=\operatorname{dim}_{k} \mathscr{L}_{2}\left(2 d-2 ;(d-1)^{2}, 0^{3}\right)=1,
$$

as desired.

If $n \geq 4$, then we conclude by induction using (6-2). Thus, Claim 1 is shown.

Next we consider the right-hand side of inequality (6-1). Taking into account Claim 1, we see that $R / I$ fails the WLP, once we have shown the following:

Claim 2. $\operatorname{dim}_{k}[R / I]_{n d-n} \leq \operatorname{dim}_{k}[R / I]_{n d-n-1}$.

Proof. We use induction on $n \geq 3$. Let $A=R /\left\langle x_{1}^{d}, \ldots, x_{2 n}^{d}\right\rangle$. Assume $n=3$. Lemma 3.7 provides

$\operatorname{dim}_{k}[R / I]_{3 d-3}-\operatorname{dim}_{k}[R / I]_{3 d-4}$

$$
\begin{aligned}
& =h_{A}(3 d-3)-h_{A}(2 d-3)-h_{A}(3 d-4)+h_{A}(2 d-4) \\
& =\left(\begin{array}{c}
3 d+1 \\
4
\end{array}\right)-7\left(\begin{array}{c}
2 d+1 \\
4
\end{array}\right)+21\left(\begin{array}{c}
d+1 \\
4
\end{array}\right) \\
& =-\frac{1}{12} d(d-2)\left(5 d^{2}+2 d+5\right) \\
& \leq 0
\end{aligned}
$$

as desired.

Let $n \geq 4$. By Lemma 3.7, Claim 2 can be rewritten as

$$
h_{A}(n d-n)-h_{A}(n d-n-1) \leq h_{A}(n d-n-d)-h_{A}(n d-n-d-1) .
$$


Consider now the ring $B=k\left[x_{1}, \ldots, x_{2 n-1}\right] /\left\langle x_{1}^{d}, \ldots, x_{2 n-1}^{d}\right\rangle$. Then $A \cong B \otimes_{k}$ $k[x] /\left(x^{d}\right)$, which implies

$$
h_{A}(j)=h_{B}(j)+h_{B}(j-1)+\cdots+h_{B}(j-(d-1)) .
$$

Thus, the last inequality becomes

$$
h_{B}(n d-n)-h_{B}(n d-n-d) \leq h_{B}(n d-n-d)-h_{B}(n d-n-2 d) .
$$

The Hilbert function of $B$ is symmetric about $\frac{1}{2}(2 n-1)(d-1)$, so $h_{B}(n d-n)=$ $h_{B}(n d-n-d+1)$. Thus, we have to show

$$
h_{B}(n d-n-d+1)-h_{B}(n d-n-d) \leq h_{B}(n d-n-d)-h_{B}(n d-n-2 d) .
$$

To this end, put $C=k\left[x_{1}, \ldots, x_{2 n-2}\right] /\left\langle x_{1}^{d}, \ldots, x_{2 n-2}^{d}\right\rangle$. Then $B \cong C \otimes_{k} k[x] /\left(x^{d}\right)$. Hence, using a relation similar to (6-4), Claim 2 follows, once we have shown

$$
\begin{aligned}
& h_{C}(n d-n-d+1)-h_{C}(n d-n-2 d+1) \\
& \quad \leq h_{C}(n d-n-d)+h_{C}(n d-n-d)+\cdots+h_{C}(n d-n-2 d+1) \\
& -\left[h_{C}(n d-n-2 d)+h_{C}(n d-n-2 d-1)+\cdots+h_{C}(n d-n-3 d+1)\right]
\end{aligned}
$$

Our induction hypothesis (see inequality (6-3)) provides

$h_{C}(n d-n-d+1)-h_{C}(n d-n-d) \leq h_{C}(n d-n-2 d+1)-h_{C}(n d-n-2 d)$.

Since the Hilbert function of $C$ is unimodal with peak in degree $(n-1)(d-1)$, we have the estimates:

$$
\begin{aligned}
h_{C}(n d-n-d)-h_{C}(n d-n-2 d & +1) \\
& \leq h_{C}(n d-n-d)-h_{C}(n d-n-2 d-1), \\
0 & \leq h_{C}(n d-n-d-1)-h_{C}(n d-n-2 d-2), \\
& \vdots \\
0 & \leq h_{C}(n d-n-2 d)-h_{C}(n d-n-3 d-1) .
\end{aligned}
$$

Adding the last inequalities, we get the desired inequality (6-5), which completes the proof of Claim 2, and we are done.

Remark 6.2. Theorem 6.1 proves half of Conjecture 5.5.2 in [Harbourne et al. 2011] - namely, the case when the number of variables is even.

Remark 6.3. In [Harbourne et al. 2011, Theorem 5.2.2], Harbourne, Schenck and Seceleanu have recently given an alternative proof of the above theorem for $d \gg 0$.

Claim 2 can be restated as a result about the growth of the coefficients of a certain univariate polynomial. 
Proposition 6.4. Let $n \geq 3$ and $d \geq 1$ be integers. For the univariate polynomial

$$
a_{0}+a_{1} z+\cdots+a_{2 n d} z^{2 n d}:=\left(1+z+\cdots+z^{d}\right)^{2 n},
$$

we have $a_{n d}-a_{n d-1} \leq a_{n d-d-1}-a_{n d-d-2}$.

Proof. Note that $a_{i}$ is the Hilbert function of $k\left[x_{1}, \ldots, x_{2 n}\right] /\left\langle x_{1}^{d+1}, \ldots, x_{2 n}^{d+1}\right\rangle$ in degree $i$. Hence inequality (6-3) establishes the claim.

We now turn to an odd number of variables. We are not able to give a result as comprehensive as that of an even number of variables, and we only consider the case of seven variables.

Theorem 6.5. Let $L \in R=k\left[x_{1}, \ldots, x_{7}\right]$ be a general linear form, and let $I=$ $\left\langle x_{1}^{d}, \ldots, x_{7}^{d}, L^{d}\right\rangle$. If $d=2$ then the ring $R / I$ has the WLP. If $d \geq 4$ then $R / I$ fails the WLP.

Proof. If $d=2$, we have verified on CoCoA that $R / I$ has the WLP. In fact, CoCoA has also given the result that when $d=3, R / I$ fails to have the WLP because of the failure of injectivity. However, a computer verification is not enough to show the failure of the WLP, since it is impossible to justify that the linear forms are "general enough". We conjecture that WLP also fails for $d=3$.

We now assume that $d \geq 4$. We will show the failure of surjectivity in a suitable degree. Let $\ell \in R$ be a general linear form. Let $j=\left\lfloor\frac{17}{5}(d-1)\right\rfloor$. We want to compute $\operatorname{dim}_{k}[R /(I, \ell)]_{j}=\operatorname{dim}_{k} \mathscr{L}_{5}\left(j ;(j+1-d)^{8}\right)$, and in particular, to show that this dimension is nonzero. Using Lemma 3.5 four times we get

$$
\begin{aligned}
\operatorname{dim}_{k}[R /(I, \ell)]_{j} \\
\quad=\operatorname{dim}_{k} \mathscr{L}_{5}\left(-j+6(d-1) ;(j+1-d)^{2},(-j+5(d-1))^{6}\right) \\
\quad=\operatorname{dim}_{k} \mathscr{L}_{5}\left(-3 j+12(d-1) ;(-j+5(d-1))^{4},(-3 j+11(d-1))^{4},\right) \\
\quad=\operatorname{dim}_{k} \mathscr{L}_{5}\left(-5 j+18(d-1) ;(-3 j+11(d-1))^{6},(-5 j+17(d-1))^{2}\right) \\
\quad=\operatorname{dim}_{k} \mathscr{L}_{5}\left(-7 j+24(d-1) ;(-5 j+17(d-1))^{8}\right) .
\end{aligned}
$$

This computation is correct and has a chance of resulting in a nonempty linear system if $0 \leq-5 j+17(d-1)<-7 j+24(d-1)$, which is true since $j \leq \frac{17}{5}(d-1)$.

Thus, we distinguish five cases, where $e$ is an integer:

Case 1: $d-1=5 e$, thus $j=17 e$.

Case 2: $d-1=5 e+1$, thus $j=17 e+3$.

Case 3: $d-1=5 e+2$, thus $j=17 e+6$.

Case 4: $d-1=5 e+3$, thus $j=17 e+10$.

Case 5: $d-1=5 e+4$, thus $j=17 e+13$. 
The computation (6-6) then shows that $\operatorname{dim}_{k}[R /(I, \ell)]_{j}$ equals $\operatorname{dim}_{k} \mathscr{L}_{5}\left(e ; 0^{8}\right)$ in Case $1, \operatorname{dim}_{k} \mathscr{L}_{5}\left(e+3 ; 2^{8}\right)$ in Case $2, \operatorname{dim}_{k} \mathscr{L}_{5}\left(e+6 ; 4^{8}\right)$ in Case $3, \operatorname{dim}_{k} \mathscr{L}_{5}\left(e+2 ; 1^{8}\right)$ in Case 4 , and $\operatorname{dim}_{k} \mathscr{L}_{5}\left(e+5 ; 3^{8}\right)$ in Case 5 . It is clear that these linear systems are not empty if $e \geq 0$, thus $d \geq 1$.

To prove failure of the WLP in degree $j$ it remains to check that the expected dimension is zero. Using Lemma 3.7, we obtain

$$
\begin{aligned}
\operatorname{dim}_{k}[R / I]_{j}-\operatorname{dim}_{k}[R / I]_{j-1} & \\
= & \left(\begin{array}{c}
j+5 \\
5
\end{array}\right)-8\left(\begin{array}{c}
j+5-d \\
5
\end{array}\right)+28\left(\begin{array}{c}
j+5-2 d \\
5
\end{array}\right)-56\left(\begin{array}{c}
j+5-3 d \\
5
\end{array}\right) .
\end{aligned}
$$

Notice that the last binomial coefficient is zero if $d \leq 10$, while the third one is zero for $d \leq 2$. However, the computations of the polynomials below are not affected. Distinguishing the five cases above, this dimension times 5 ! equals

Case 1: $-101995 e^{5}-69925 e^{4}-15975 e^{3}+565 e^{2}+730 e+120$

Case 2: $-101995 e^{5}-139850 e^{4}-60225 e^{3}-1330 e^{2}+5080 e+960$

Case 3: $-101995 e^{5}-209775 e^{4}-133975 e^{3}-8145 e^{2}+19730 e+5040$

Case 4: $-101995 e^{5}-359875 e^{4}-499175 e^{3}-336365 e^{2}-107910 e-12600$

Case 5: $-5(e+1)\left(20399 e^{4}+65561 e^{3}+74044 e^{2}+32716 e+3840\right)$

Clearly the first three polynomials are negative if $e \geq 1$, and the last two whenever $e \geq 0$. Thus the expected dimension is zero whenever $d \geq 4$. In particular, we have shown the failure of surjectivity (in particular the failure of the WLP) for $d \geq 4$.

In applying the approach of Theorem 6.5 to the general case, we were able to mimic the choice of $j$, as well as the main details of the proof that $\operatorname{dim}[R /(I, \ell)]_{j}>$ 0 . However, a proof of the required inequality to verify that it is surjectivity rather than injectivity that fails eluded us. Based on experiments with CoCoA, we end with the following conjecture (also to complete the case of seven variables). Notice that the case $d=2$ has the WLP in seven variables (as noted above).

Conjecture 6.6. Let $R=k\left[x_{1}, \ldots, x_{2 n+1}\right]$, where $n \geq 4$. Let $L \in R$ be a general linear form, and let $I=\left\langle x_{1}^{d}, \ldots, x_{2 n+1}^{d}, L^{d}\right\rangle$. Then the ring $R / I$ fails the WLP if and only if $d>1$. Furthermore, if $n=3$ then $R / I$ fails the WLP when $d=3$.

\section{Acknowledgments}

Shortly before the end of the writing of this paper we were informed that B. Harbourne, H. Schenck and A. Seceleanu were able to prove Theorem 6.1 for $d \gg 0$. We are very grateful to $\mathrm{H}$. Schenk for sending their paper to us, and for the helpful discussions we have had. We also thank M. Boij for interesting and useful discussions, and the referees for helpful comments. 


\section{References}

[Anick 1986] D. J. Anick, "Thin algebras of embedding dimension three", J. Algebra 100:1 (1986), 235-259. MR 88d:13016a Zbl 0588.13013

[Ardila and Postnikov 2010] F. Ardila and A. Postnikov, "Combinatorics and geometry of power ideals”, Trans. Amer. Math. Soc. 362:8 (2010), 4357-4384. MR 2011g:05322 Zbl 1226.05019

[Boij et al. 2012] M. Boij, J. Migliore, R. Miró-Roig, U. Nagel, and F. Zanello, On the shape of a pure O-sequence, Mem. Amer. Math. Soc. 1024, Amer. Math. Soc., Providence, RI, 2012.

[Brenner and Kaid 2007] H. Brenner and A. Kaid, "Syzygy bundles on $\mathbb{P}^{2}$ and the weak Lefschetz property”, Illinois J. Math. 51:4 (2007), 1299-1308. MR 2009j:13012 Zbl 1148.13007

[Brenner and Kaid 2011] H. Brenner and A. Kaid, "A note on the weak Lefschetz property of monomial complete intersections in positive characteristic", Collect. Math. 62:1 (2011), 85-93. MR 2012c:13031 Zbl 1221.13019

[Catalisano et al. 1999] M. V. Catalisano, P. Ellia, and A. Gimigliano, "Fat points on rational normal curves”, J. Algebra 216:2 (1999), 600-619. MR 2000d:13025 Zbl 0940.14035

[Chandler 2005] K. A. Chandler, "The geometric interpretation of Fröberg-Iarrobino conjectures on infinitesimal neighbourhoods of points in projective space", J. Algebra 286:2 (2005), 421-455. MR 2005k:14008 Zbl 1067.14047

[Chandler 2007] K. A. Chandler, "Examples and counterexamples on the conjectured Hilbert function of multiple points", pp. 13-31 in Algebra, geometry and their interactions (Notre Dame, IN, 2005), edited by A. Corso et al., Contemp. Math. 448, Amer. Math. Soc., Providence, RI, 2007. MR 2009c:13036 Zbl 1067.14047

[CoCoA 2009] CoCoA, "CoCoA: a system for doing computations in commutative algebra", 2009, Available at http://cocoa.dima.unige.it.

[Cook and Nagel 2009] D. Cook II and U. Nagel, "The weak Lefschetz property, monomial ideals, and lozenges", preprint, 2009. To appear in Illinois J. Math. arXiv 0909.3509

[Cook and Nagel 2011] D. Cook II and U. Nagel, "Enumerations deciding the weak Lefschetz property", preprint, 2011. arXiv 1105.6062

[De Volder and Laface 2007] C. De Volder and A. Laface, "On linear systems of $\mathbb{P}^{3}$ through multiple points", J. Algebra 310:1 (2007), 207-217. MR 2008b:14009 Zbl 1113.14036

[Di Rocco 1996] S. Di Rocco, " $k$-very ample line bundles on del Pezzo surfaces", Math. Nachr. 179 (1996), 47-56. MR 97h:14007 Zbl 0870.14031

[Dumnicki 2009] M. Dumnicki, "An algorithm to bound the regularity and nonemptiness of linear systems in $\mathbb{P}^{n}$ ”, J. Symbolic Comput. 44:10 (2009), 1448-1462. MR 2010i:14108 Zbl 1205.14006

[Emsalem and Iarrobino 1995] J. Emsalem and A. Iarrobino, "Inverse system of a symbolic power. I”, J. Algebra 174:3 (1995), 1080-1090. MR 96i:13017 Zbl 0842.14002

[Fröberg 1985] R. Fröberg, “An inequality for Hilbert series of graded algebras”, Math. Scand. 56:2 (1985), 117-144. MR 87f:13022 Zbl 0582.13007

[Geramita et al. 2009] A. V. Geramita, B. Harbourne, and J. Migliore, "Classifying Hilbert functions of fat point subschemes in $\mathbb{P}^{2}$ ", Collect. Math. 60:2 (2009), 159-192. MR 2010g:13025 Zbl 1186.14008

[Harbourne et al. 2011] B. Harbourne, H. Schenck, and A. Seceleanu, "Inverse systems, GelfandTsetlin patterns and the weak Lefschetz property”, J. Lond. Math. Soc. (2) 84:3 (2011), 712-730. MR 2855798 Zbl 05987713 
[Harima et al. 2003] T. Harima, J. Migliore, U. Nagel, and J. Watanabe, "The weak and strong Lefschetz properties for Artinian K-algebras”, J. Algebra 262:1 (2003), 99-126. MR 2004b:13001 Zbl 1018.13001

[Iarrobino 1997] A. Iarrobino, "Inverse system of a symbolic power, III: Thin algebras and fat points", Compositio Math. 108:3 (1997), 319-356. MR 98k:13017 Zbl 0899.13016

[Laface and Ugaglia 2006] A. Laface and L. Ugaglia, "On a class of special linear systems of $\mathbb{P}^{3}$ ", Trans. Amer. Math. Soc. 358:12 (2006), 5485-5500. MR 2007e:14009 Zbl 1160.14003

[Li and Zanello 2010] J. Li and F. Zanello, "Monomial complete intersections, the weak Lefschetz property and plane partitions", Discrete Mathematics 310:24 (2010), 3558-3570. MR 2012a:13032 Zbl 1202.13013

[Migliore and Miró-Roig 2003] J. Migliore and R. M. Miró-Roig, "Ideals of general forms and the ubiquity of the weak Lefschetz property", J. Pure Appl. Algebra 182:1 (2003), 79-107. MR 2004c: 13027 Zbl 1041.13011

[Migliore et al. 2011] J. C. Migliore, R. M. Miró-Roig, and U. Nagel, "Monomial ideals, almost complete intersections and the weak Lefschetz property", Trans. Amer. Math. Soc. 363:1 (2011), 229-257. MR 2011i:13020

[Nagata 1960] M. Nagata, "On the fourteenth problem of Hilbert", pp. 459-462 in Proc. Internat. Congress Math. 1958, Cambridge Univ. Press, New York, 1960. MR 22 \#6851 Zbl 0127.26302

[Reid et al. 1991] L. Reid, L. G. Roberts, and M. Roitman, “On complete intersections and their Hilbert functions”, Canad. Math. Bull. 34:4 (1991), 525-535. MR 93b:13025 Zbl 0757.13005

[Schenck and Seceleanu 2010] H. Schenck and A. Seceleanu, "The weak Lefschetz property and powers of linear forms in $\mathbb{K}[x, y, z]$ ”, Proc. Amer. Math. Soc. 138:7 (2010), 2335-2339. MR 2011b: 13042 Zbl 1192.13013

[Stanley 1980] R. P. Stanley, "Weyl groups, the hard Lefschetz theorem, and the Sperner property", SIAM J. Algebraic Discrete Methods 1:2 (1980), 168-184. MR 82j:20083 Zbl 0502.05004

[Sturmfels and Xu 2010] B. Sturmfels and Z. Xu, "Sagbi bases of Cox-Nagata rings", J. Eur. Math. Soc. (JEMS) 12:2 (2010), 429-459. MR 2011f:13009 Zbl 1202.14053

[Watanabe 1987] J. Watanabe, "The Dilworth number of Artinian rings and finite posets with rank function", pp. 303-312 in Commutative algebra and combinatorics (Kyoto, 1985), edited by M. Nagata and H. Matsumura, Adv. Stud. Pure Math. 11, North-Holland, Amsterdam, 1987. MR 89k: 13015 Zbl 0648.13010

[Zanello 2006] F. Zanello, “A non-unimodal codimension 3 level $h$-vector”, J. Algebra 305:2 (2006), 949-956. MR 2007h:13026 Zbl 1110.13012

Communicated by Craig Huneke

Received 2010-09-13 Revised 2011-06-09 Accepted 2011-07-19

migliore.1@nd.edu

Department of Mathematics, University of Notre Dame, 255 Hurley Hall, Notre Dame, IN 46556, United States

miro@ub.edu

Facultat de Matemàtiques,

Departament d'Algebra i Geometria, University of Barcelona, Gran Via de les Corts Catalanes 585, 08007 Barcelona, Spain

uwe.nagel@uky.edu

Department of Mathematics, University of Kentucky, 715 Patterson Ofiice Tower, Lexington, KY 40506-0027, United States 


\section{Algebra \& Number Theory}

msp.berkeley.edu/ant

\section{EDITORS}

MANAGING EDITOR

Bjorn Poonen

Massachusetts Institute of Technology

Cambridge, USA

\author{
EDITORIAL BOARD CHAIR \\ David Eisenbud \\ University of California \\ Berkeley, USA
}

\section{BOARD OF EDITORS}

Georgia Benkart

Dave Benson

Richard E. Borcherds

John H. Coates

J-L. Colliot-Thélène

Brian D. Conrad

Hélène Esnault

Hubert Flenner

Edward Frenkel

Andrew Granville

Joseph Gubeladze

Ehud Hrushovski

Craig Huneke

Mikhail Kapranov

Yujiro Kawamata

János Kollár

Yuri Manin

Barry Mazur

Philippe Michel

Susan Montgomery
University of Wisconsin, Madison, USA

University of Aberdeen, Scotland

University of California, Berkeley, USA

University of Cambridge, UK

CNRS, Université Paris-Sud, France

University of Michigan, USA

Universität Duisburg-Essen, Germany

Ruhr-Universität, Germany

University of California, Berkeley, USA

Université de Montréal, Canada

San Francisco State University, USA

Hebrew University, Israel

University of Kansas, USA

Yale University, USA

University of Tokyo, Japan

Princeton University, USA

Northwestern University, USA

Harvard University, USA

École Polytechnique Fédérale de Lausanne

University of Southern California, USA
Shigefumi Mori

Raman Parimala

Jonathan Pila

Victor Reiner

Karl Rubin

Peter Sarnak

Joseph H. Silverman

Michael Singer

Ronald Solomon

Vasudevan Srinivas

J. Toby Stafford

Bernd Sturmfels

Richard Taylor

Ravi Vakil

Michel van den Bergh

Marie-France Vignéras

Kei-Ichi Watanabe

Andrei Zelevinsky

Efim Zelmanov
RIMS, Kyoto University, Japan

Emory University, USA

University of Oxford, UK

University of Minnesota, USA

University of California, Irvine, USA

Princeton University, USA

Brown University, USA

North Carolina State University, USA

Ohio State University, USA

Tata Inst. of Fund. Research, India

University of Michigan, USA

University of California, Berkeley, USA

Harvard University, USA

Stanford University, USA

Hasselt University, Belgium

Université Paris VII, France

Nihon University, Japan

Northeastern University, USA

University of California, San Diego, USA

\section{PRODUCTION}

contact@msp.org

Silvio Levy, Scientific Editor

See inside back cover or www.jant.org for submission instructions.

The subscription price for 2012 is US \$175/year for the electronic version, and \$275/year (+\$40 shipping outside the US) for print and electronic. Subscriptions, requests for back issues from the last three years and changes of subscribers address should be sent to Mathematical Sciences Publishers, Department of Mathematics, University of California, Berkeley, CA 94720-3840, USA.

Algebra \& Number Theory (ISSN 1937-0652) at Mathematical Sciences Publishers, Department of Mathematics, University of California, Berkeley, CA 94720-3840 is published continuously online. Periodical rate postage paid at Berkeley, CA 94704, and additional mailing offices.

ANT peer review and production are managed by EditFLOW ${ }^{\circledR}$ from Mathematical Sciences Publishers.

PUBLISHED BY

mathematical sciences publishers

http://msp.org/

A NON-PROFIT CORPORATION

Typeset in IAT $_{\mathrm{E}} \mathrm{X}$

Copyright (C2012 by Mathematical Sciences Publishers 


\section{Algebra \& Number Theory}

Volume $6 \quad$ No. $3 \quad 2012$

The image of complex conjugation in $l$-adic representations associated to automorphic forms

\section{RICHARD TAYLOR}

Betti numbers of graded modules and the multiplicity conjecture in the

non-Cohen-Macaulay case

\section{MATS BOIJ and JONAS SÖDERBERG}

$\mathscr{L}$-invariants and Shimura curves

SAMIT DAsGupta and MATTHEW GREENBERG

On the weak Lefschetz property for powers of linear forms

Juan C. Migliore, Rosa M. Miró-Roig and Uwe Nagel

Resonance equals reducibility for $A$-hypergeometric systems

Mathias Schulze and Uli WaLther

The Chow ring of double EPW sextics

ANDREA FERRETTI

A finiteness property of graded sequences of ideals

Mattias JONSSON and Mircea MustaȚĂ

On unit root formulas for toric exponential sums

Alan Adolphson and SteVen Sperber

Symmetries of the transfer operator for $\Gamma_{0}(N)$ and a character deformation of the 